\title{
Derived McKay correspondence via pure-sheaf transforms
}

\section{Timothy Logvinenko}

the date of receipt and acceptance should be inserted later - (C) Springer-Verlag 2007

\begin{abstract}
In most cases where it has been shown to exist the derived McKay correspondence $D(Y) \stackrel{\sim}{\longrightarrow} D^{G}\left(\mathbb{C}^{n}\right)$ can be written as a Fourier-Mukai transform which sends point sheaves of the crepant resolution $Y$ to pure sheaves in $D^{G}\left(\mathbb{C}^{n}\right)$. We give a sufficient condition for $E \in$ $D^{G}\left(Y \times \mathbb{C}^{n}\right)$ to be the defining object of such a transform. We use it to construct the first example of the derived McKay correspondence for a non-projective crepant resolution of $\mathbb{C}^{3} / G$. Along the way we extract more geometrical meaning out of the Intersection Theorem and learn to compute $\theta$-stable families of $G$-constellations and their direct transforms.
\end{abstract}

Mathematics Subject Classification (2000): <14E15, 14J10, 18E30>

\section{Introduction}

It was observed by McKay in [McK80] that the representation graph (better known now as the McKay quiver) of a finite subgroup $G$ of $\mathrm{SL}_{2}(\mathbb{C})$ is the Coxeter graph of one of the affine Lie algebras of type ADE, while the configuration of irreducible exceptional divisors on the minimal resolution $Y$ of $\mathbb{C}^{2} / G$ is dual to the Coxeter graph of the finite-dimensional Lie algebra of the same type. It followed that the subgraph of nontrivial irreducible representations coincided with the graph of irreducible exceptional divisors. This led Gonzales-Sprinberg and Verdier in [GSV83] to construct an isomorphism of the $G$-equivariant $K$ theory of $\mathbb{C}^{2}$ to the $K$-theory of $Y$, which induced naturally a choice of such bijection. This became known as the (classical) McKay correspondence.

In [Rei97] M.Reid proposed that the $K$-theory isomorphism might lift to the level of derived categories. It became known as the derived McKay correspondence conjecture:

Conjecture 1. Let $G$ be a finite subgroup of $\mathrm{SL}_{n}(\mathbb{C})$ and let $Y$ be a crepant resolution of $\mathbb{C}^{n} / G$, if one exists. Then

$$
D(Y) \stackrel{\sim}{\longrightarrow} D^{G}\left(\mathbb{C}^{n}\right)
$$

TIMOTHY LOGVINENKO

Department of Mathematics,

KTH,

Stockholm, SE-100 44, Sweden 
where $D(Y)$ and $D^{G}\left(\mathbb{C}^{n}\right)$ are bounded derived categories of coherent sheaves on $Y$ and of $G$-equivariant coherent sheaves on $\mathbb{C}^{n}$, respectively.

To date and to the extent of our knowledge this conjecture has been settled for the following situations:

1. $G \subset \mathrm{SL}_{2,3}(\mathbb{C}) ; Y$ the distinguished crepant resolution $G$-Hilb;

([KV98], Theorem 1.4; [BKR01], Theorem 1.1).

2. $G \subset \mathrm{SL}_{3}(\mathbb{C})$ abelian; $Y$ any projective crepant resolution; ([CI04], Theorem 1.1).

3. $G \subset \mathrm{SL}_{n}(\mathbb{C})$ abelian; $Y$ any projective crepant resolution; ([Kaw05], special case of Theorem 4.2).

4. $G \subset \mathrm{Sp}_{2 n}(\mathbb{C}) ; Y$ any symplectic (crepant) resolution; ([BK04], Theorem 1.1).

In the case 3 the construction is not direct and it isn't clear what form does the equivalence (1.1) take, but in each of the cases 1, 2 and 4, the equivalence (1.1) is constructed directly and we observe that the constructed functor sends point sheaves $\mathcal{O}_{y}$ of $Y$ to pure sheaves (i.e. complexes with cohomologies concentrated in degree zero) in $D^{G}\left(\mathbb{C}^{n}\right)$. Another property (cf. though [Orl97], Theorem 2.18) that these functors share is that each can be written as a Fourier-Mukai transform $\Phi_{E}\left(-\otimes \rho_{0}\right)$ (see Def. 3) for some object $E \in D^{G}\left(Y \times \mathbb{C}^{n}\right)$.

A straightforward application (Prop. 3) of the established machinery of FourierMukai transforms shows that if an equivalence (1.1) is a Fourier-Mukai transform $\Phi_{E}\left(-\otimes \rho_{0}\right)$ which sends point sheaves to pure sheaves, then its defining object $E$ is itself a pure sheaf. Moreover, the fibers of $E$ over $Y$ have to be simple $\left(G-\operatorname{End}_{\mathbb{C}^{n}}\left(E_{\mid y}\right)=\mathbb{C}\right.$ for all $\left.y \in Y\right)$, orthogonal in all degrees $(G$ $\operatorname{Ext}_{\mathbb{C}^{n}}^{i}\left(E_{\mid y_{1}}, E_{\mid y_{2}}\right)=0$ if $\left.y_{1} \neq y_{2}\right)$ and the Kodaira-Spencer maps have to be isomorphisms.

Let $Y$ now be any irreducible separated scheme of finite type over $\mathbb{C}$. A gnat-family $\mathcal{F}$ on $Y$ is a coherent $G$-sheaf on $Y \times \mathbb{C}^{n}$, flat over $Y$, such that for any $y \in Y$ the fiber $\mathcal{F}_{\mid y}$ of $\mathcal{F}$ is a $G$-constellation supported on a single $G$-orbit. That is, $\mathcal{F}_{\mid y}$ is a finite length coherent $G$-sheaf on $\mathbb{C}^{n}$ whose support is a single $G$-orbit and whose global sections have $G$-representation structure of the regular representation. Such family $\mathcal{F}$ has a well-defined Hilbert-Chow morphism $\pi_{\mathcal{F}}$ : $Y \rightarrow \mathbb{C}^{n} / G$, it sends any $y \in Y$ to the $G$-orbit that $\mathcal{F}_{\mid y}$ is supported on (Prop. 2). Let $Y$ and $\mathcal{F}$ be any such for which $\pi_{\mathcal{F}}$ is birational and proper. In this paper we give a sufficient condition for the functor $\Phi_{\mathcal{F}}\left(-\otimes \rho_{0}\right)$ to be an equivalence (1.1). Notable, in the view of Prop. 3, is that this condition only asks for the non-orthogonality locus of $\mathcal{F}$ to be of high enough codimension. The simplicity of $\mathcal{F}$ and the Kodaira-Spencer maps being isomorphisms follow automatically:

Theorem 1. Let $G$ be a finite subgroup of $\mathrm{SL}_{n}(\mathbb{C})$. Let $Y$ be an irreducible separated scheme of finite type over $\mathbb{C}$ and $\mathcal{F}$ be a gnat-family on $Y$. Assume $Y$ and $\mathcal{F}$ such that the Hilbert-Chow morphism $\pi_{\mathcal{F}}$ is birational and proper. 
If for every $0 \leq k<(n+1) / 2$, the codimension of the subset

$$
\mathrm{N}_{k}=\overline{\left\{\left(y_{1}, y_{2}\right) \in Y \times Y \backslash \Delta \mid G-\operatorname{Ext}_{\mathbb{C}^{n}}^{k}\left(\mathcal{F}_{\mid y_{1}}, \mathcal{F}_{\mid y_{2}}\right) \neq 0\right\}}
$$

in $Y \times Y$ is at least $n+1-2 k$, then the functor $\Phi_{\mathcal{F}}\left(-\otimes \rho_{0}\right)$ is an equivalence of categories $D(Y) \stackrel{\sim}{\longrightarrow} D^{G}\left(\mathbb{C}^{n}\right)$.

Once $\Phi_{\mathcal{F}}\left(-\otimes \rho_{0}\right)$ is known to be an equivalence usual methods ([Rob98], Theorem 6.2.2 and [BKR01], Lemma 3.1) apply to show that $Y$ is non-singular and $\pi_{\mathcal{F}}$ is crepant. The set $N_{k}$ in (1.2) can be thought of as the locus of the degree $k$ non-orthogonality in $\mathcal{F}$.

Our proof of Theorem 1 is based on the ideas introduced in [BO95] and [BKR01], particularly on the Intersection Theorem trick introduced in the latter. However, not wishing to restrict ourselves to just quasi-projective schemes necessitates more work in applying the Intersection Theorem. This is done in Section 2, which is a self-contained piece of abstract derived category theory for a locally noetherian scheme $X$. There we propose a generalisation of the concept of the homological dimension of $E \in D_{\text {coh }}^{b}(X)$ which we call Toramplitude, and use it to show that the inequality

$$
\text { hom. dim. } E \geq \operatorname{codim}_{X} \operatorname{Supp} E
$$

of [BM02], Corollary 5.5 refines to

$$
\text { Tor-amp } E \geq \operatorname{codim}_{X} \operatorname{Supp} E+\text { coh-amp } E \text {. }
$$

Other notable points of our proof of Theorem 1 are a different approach to Grothendieck duality when constructing the left adjoint to $\Phi_{\mathcal{F}}\left(-\otimes \rho_{0}\right)$ and an application of [Log06], Prop. 1.5 which states that outside the exceptional set of $Y$ any gnat-family has to be locally isomorphic to the universal family of $G$-clusters. The latter is everywhere simple and its Kodaira-Spencer maps are isomorphisms. Then the locus of points of $Y$ where objects of $\mathcal{F}$ are not simple or the Kodaira-Spencer map isn't an isomorphism turns out to have too high a codimension to exist at all.

The question of an existence of a derived McKay correspondence which sends point sheaves to pure sheaves is thus reduced to that of an existence of a gnat-family satisfying the non-orthogonality condition of Theorem 1 . This is particularly relevant whenever $G$ is abelian, for then all the gnat-families on a given resolution $Y \rightarrow \mathbb{C}^{n} / G$ had been classified and their number was shown to be finite and non-zero ([Log06], Theorem 4.1).

When $n=3$, Theorem 1 reduces to:

Corollary 1. Let $G$ be a finite subgroup of $\mathrm{SL}_{3}(\mathbb{C})$. Let $Y, \mathcal{F}$ and $\pi_{\mathcal{F}}$ be as in Theorem 1. Let $E_{1}, \ldots, E_{k}$ be the irreducible exceptional surfaces of $\pi_{\mathcal{F}}$. Then if general points of any surface $E_{i}$ are orthogonal in degree 0 in $\mathcal{F}$ to general points of any surface $E_{j}$ (including case $j=i$ ) and of any curve $E_{l} \cap E_{m}$, then $\Phi_{\mathcal{F}}\left(-\otimes \rho_{0}\right)$ is an equivalence of categories. 
By a general point of an intersection of $k$ exceptional surfaces we mean a point that doesn't lie on an intersection of any $k+1$ exceptional surfaces.

In Section 4 we show how to compute the degree 0 non-orthogonality locus of a gnat-family. We use this in Section 5 to give following application of Corollary 1: for $G$ the abelian subgroup of $\mathrm{SL}_{3}(\mathbb{C})$ known as $\frac{1}{6}(1,1,4) \oplus \frac{1}{2}(1,0,1)$ (see Section 5.1) and for $Y$ a certain non-projective crepant resolution of $\mathbb{C}^{3} / G$ (see Section 5.2) we construct a gnat-family $\mathcal{F}$ on $Y$ which satisfies the condition in Corollary 1. This gives the first example of the derived McKay correspondence for a non-projective crepant resolution of $\mathbb{C}^{3} / G$.

It also leads to an important observation: the properties that $\mathcal{F}$ must then possess in view of Proposition 3 imply that $Y$ is a fine moduli space of $G$ constellations, representing the functor of all gnat-families whose members (fibres over closed points) are isomorphic to members of $\mathcal{F}$. At present the only moduli functors known for $G$-constellations come from the notion of $\theta$-stability. Their fine moduli spaces $M$ (cf. [CI04]) are constructed via the method introduced by King in [Kin94]. However, $Y$ can't be one of $M$ as these are all, due to the GIT nature of their construction in [Kin94], projective over $\mathbb{C}^{n} / G$. This raises the question as to whether there could exist a more general notion of 'stability', related perhaps to Bridgeland-Douglas stability [Bri02], which would allow for functors with non-projective moduli spaces.

Acknowledgements: The author would like to express his gratitude to S. Mukai, D. Kaledin, D. Orlov and A. Bondal for useful discussions while the paper was written, to A. Craw for observing a crucial link with the work in [CMT05a], [CMT05b] which inspired the Proposition 6 and to A. King and an anonymous referee for many helpful comments on the first draft. The paper was originally completed during the author's stay at RIMS, Kyoto, and he would like to thank everyone at the institute for their hospitality. A substantial revision was then carried out during the author's stay at Mittag-Leffler Institute/KTH, Stockholm, and he would like to thank them also.

\section{Cohomological and Tor amplitudes}

We clarify terminology and introduce notation. By a point of a scheme we mean both a closed and non-closed point unless specifically mentioned otherwise. Given a point $x$ on a scheme $X$ we write $\left(\mathcal{O}_{x}, \mathfrak{m}_{x}\right)$ for the local ring of $x, \mathbf{k}(x)$ for the residue field $\mathcal{O}_{x} / \mathfrak{m}_{x}$ and $\iota_{x}$ for the point-scheme inclusion Spec $\mathbf{k}(x) \hookrightarrow X$. Given an irreducible closed set $C \subset X$, we write $x_{C}$ for the generic point of $C$ and we sometimes write simply $\left(\mathcal{O}_{C}, \mathfrak{m}_{C}\right)$ for the local ring of $x_{C}$. All complexes are cochain complexes. Given a right (resp. left) exact functor $F$ between two abelian categories $\mathcal{A}$ and $\mathcal{B}$, we denote by $\mathbf{L} F$ (resp. $\mathbf{R} F$ ) the left (resp. right) derived functor between the appropriate derived cat- 
egories, if it exists, and by $\mathbf{L}^{i} F(\bullet)\left(\right.$ resp. $\left.\mathbf{R}^{i} F(\bullet)\right)$ the $-i$-th cohomology of $\mathbf{L} F(\bullet)($ resp. the $i$-th cohomology of $\mathbf{R} F(\bullet)$ ).

For $X$ a smooth variety the results of Lemmas 1 and 2 below have appeared in the proof of Proposition 1.5 in [BO95]. We show them to hold in a more general setting of a locally noetherian scheme.

Lemma 1. Let $X$ be a locally noetherian scheme. Let $\mathcal{F}$ be a coherent sheaf on $X$ and $C$ be an irreducible component of $\operatorname{Supp}_{X} \mathcal{F}$. Then for every point $x \in C$

$$
\mathbf{L}^{i} \iota_{x}^{*} \mathcal{F} \neq 0 \quad \text { for } 0 \leq i \leq \operatorname{codim}_{X}(C) .
$$

Proof. Recall (cf. [Mat86], §19) that if a minimal free resolution $L_{\bullet}$ of a finitely generated module $M$ for a local ring $(R, \mathfrak{m}, k)$ exists, then

$$
\operatorname{dim}_{k} \operatorname{Tor}^{i}(M, k)=\operatorname{rk} L_{i}
$$

Since $X$ is locally noetherian minimal free resolutions of $\mathcal{F}$ exist in all local rings. Write $F_{C}$ for the localisation of $\mathcal{F}$ to the local ring $\mathcal{O}_{C}$ of $x_{C}$. As $\mathbf{L}^{i} \iota_{x}^{*} \mathcal{F}=\operatorname{Tor}_{\mathcal{O}_{C}}^{i}\left(F_{C}, \mathbf{k}(x)\right)$ it suffices to prove that the length of the minimal free resolution of $F_{C}$ is at least $\operatorname{codim}_{X}(C)$.

Consider the standard filtration ([Ser00], I, §7, Theorem 1) of $F_{C}$ by submodules $0=M_{0} \subset \cdots \subset M_{n}=F_{C}$ with each $M_{i} / M_{i-1}$ isomorphic to $\mathcal{O}_{C} / \mathfrak{p}$ for some $\mathfrak{p} \in \operatorname{Supp}_{\mathcal{O}_{C}}\left(F_{C}\right)$. As the defining ideal of $C$ is minimal in $\operatorname{Supp}_{X}(\mathcal{F}), \operatorname{Supp}_{\mathcal{O}_{C}}\left(F_{C}\right)$ consists of just $\mathfrak{m}_{C}$. So each $M_{i} / M_{i-1}$ is isomorphic to $k_{C}$ and hence $F$ is a finite-length $\mathcal{O}_{C}$-module. Then by the New Intersection Theorem (e.g. [Rob98], Theorem 6.2.2) the length of the minimal resolution of $F_{C}$ is at least $\operatorname{dim} \mathcal{O}_{C}$. As $\operatorname{dim} \mathcal{O}_{C}=\operatorname{codim}_{X}(C)$ the claim follows.

Lemma 2. Let $X$ be a locally noetherian scheme. Let $\mathcal{F}$ be a coherent sheaf on $X$ of finite Tor-dimension. For any $p \in \mathbb{Z}$ define

$$
D_{p}=\left\{x \in X \mid \mathbf{L}^{i} \iota_{x}^{*} \mathcal{F} \neq 0 \text { for some } i \geq p\right\} .
$$

Then each $D_{p}$ is closed and $\operatorname{codim}_{X}\left(D_{p}\right) \geq p$.

Proof. It suffices to prove both claims for the case $X=$ Spec $R$ with $R$ noetherian. Write $F$ for $\Gamma(\mathcal{F})$. As $\mathbf{L}^{p} \iota_{x}^{*} \mathcal{F}=\operatorname{Tor}_{R}^{p}(F, \mathbf{k}(x))$ the first claim follows from the upper semicontinuity theorem ([GD63], Théorème 7.6.9).

For the second claim let $C$ be any irreducible component of $D_{p}$ and let $F_{C}$ be the localisation of $F$ to the local ring $\mathcal{O}_{C}$. Then $\operatorname{Tor}_{\mathcal{O}_{C}}^{p}\left(F_{C}, \mathbf{k}\left(x_{C}\right)\right) \neq 0$ by the defining property of $D_{p}$. We have ([Mat86], §19, Lemma 1)

$$
\operatorname{proj} \operatorname{dim}_{\mathcal{O}_{C}} F_{C}=\sup \left\{i \in \mathbb{Z} \mid \operatorname{Tor}_{\mathcal{O}_{C}}^{i}\left(F_{C}, \mathbf{k}\left(x_{C}\right)\right)\right\}
$$

hence proj $\operatorname{dim}_{\mathcal{O}_{C}} F_{C} \geq p$. By the Auslander-Buchsbaum equality we have

$$
\operatorname{depth}_{\mathcal{O}_{C}} \mathcal{O}_{C}=\operatorname{proj} \operatorname{dim}_{\mathcal{O}_{C}} F_{C}+\operatorname{depth}_{\mathcal{O}_{C}} F_{C}
$$

and thus $\operatorname{codim}_{X} C=\operatorname{dim} \mathcal{O}_{C} \geq \operatorname{depth}_{\mathcal{O}_{C}} \mathcal{O}_{C} \geq p$ as required. 
The main idea behind the proof of the following proposition we owe to Bondal and Orlov in [BO95], Proposition 1.5.

Proposition 1. Let $X$ be a locally noetherian scheme and $F \in D_{c o h}^{b}(X)$ an object of finite Tor-dimension. Denote by $\mathcal{H}^{i}$ the ith cohomology sheaf of $F$. Then for any point $x \in X$ we have

$$
-\sup \left\{i \in \mathbb{Z} \mid x \in \operatorname{Supp} \mathcal{H}^{i}\right\}=\inf \left\{j \in \mathbb{Z} \mid \mathbf{L}^{j} \iota_{x}^{*} F \neq 0\right\} .
$$

Let $C$ be an irreducible component of $\operatorname{Supp} \mathcal{H}^{l}$ for some $l$ such that also $C \nsubseteq \operatorname{Supp} \mathcal{H}^{m}$ for any $m<l$. Then

$$
\operatorname{codim}_{X} C-\inf \left\{i \in \mathbb{Z} \mid C \subseteq \operatorname{Supp} \mathcal{H}^{i}\right\}=\sup \left\{j \in \mathbb{Z} \mid \mathbf{L}^{j} \iota_{x_{C}}^{*} F \neq 0\right\} .
$$

Proof. Fix a point $x \in X$. The main ingredient of the proof is the standard spectral sequence (eg. [GM03], Proposition III.7.10) associated to the filtration of $\mathbf{L} \iota_{x}^{*} F$ by the rows of the Cartan-Eilenberg resolution of $F$ :

$$
E_{2}^{-p, q}=\mathbf{L}^{p} \iota_{x}^{*}\left(\mathcal{H}^{q}\right) \Rightarrow E_{\infty}^{q-p}=\mathbf{L}^{p-q} \iota_{x}^{*}(F) .
$$

Denote by $h$ the highest non-zero row of $E_{2}^{\bullet \bullet}$. As all rows above row $h$ and all columns to the right of column 0 in $E_{2}^{\bullet \bullet}$ consist entirely of zeroes

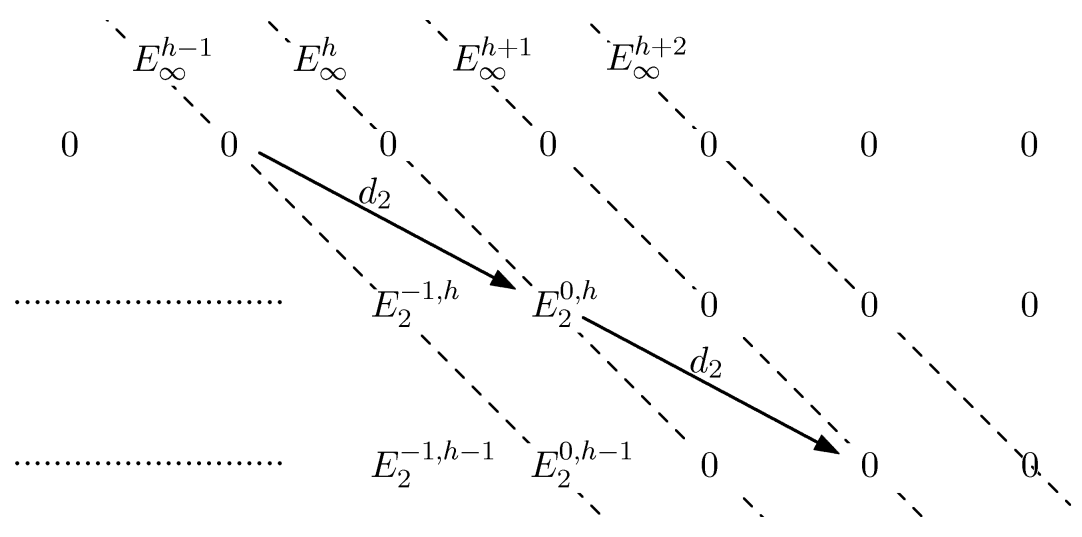

Figure 1

we conclude by inspection of the complex that $0=E_{\infty}^{n}$ for all $n>h$ and $\left.\mathcal{H}^{h}\right|_{x}=E_{2}^{0, h}=E_{\infty}^{h}=\mathbf{L}^{-h}\left(\iota_{x}^{*}(F)\right)$. This gives (2.3).

To obtain (2.4) set $x$ to be the generic point of $C$ and define $E_{\bullet}^{\bullet \bullet}$ as above. For any $m<l$ we have $C \nsubseteq$ Supp $\mathcal{H}^{m}$ and hence $\mathbf{L} \iota_{x}^{*} \mathcal{H}^{m}=0$. So all the rows of $E_{2}^{\bullet \bullet}$ below $l$ consist of zeroes. On the other hand, $C$ is an irreducible component of $\mathcal{H}^{l}$ and by Lemma 2 the set of points $y \in X$, such that there is a non-zero $\mathbf{L}^{i} \iota_{y}^{*}\left(\mathcal{H}^{l}\right)$ with $i>d$, is closed and of codimension at least $d+1$. Then this set 
can not contain $x$ for the closure of $x$ is $C$ whose codimension is $d$. Hence all columns to the left of column $-d$ in $E_{2}^{\bullet \bullet}$ consist entirely of zeroes. We conclude that $E_{\infty}^{n}=0$ for all $n>l-d$ and $\mathbf{L}^{d} \iota_{x}^{*} \mathcal{H}^{l}=E_{2}^{-d, l}=E_{\infty}^{l-d}=\mathbf{L}^{d-l} \iota_{x}^{*} F$. Thus, as $\mathbf{L}^{d} \iota_{x}^{*} \mathcal{H}^{l} \neq 0$ by Lemma 1, we obtain (2.4).

Definition 1. Let $\mathbf{A}$ be an abelian category and $E^{\bullet}$ be a cochain complex of objects of $\mathbf{A}$. Define its cohomological amplitude, denoted by coh-amp $E^{\bullet}$, to be the length of the minimal interval in $\mathbb{Z}$ containing the set

$$
\left\{i \in \mathbb{Z} \mid H^{i}\left(E^{\bullet}\right) \neq 0\right\} .
$$

If no such interval exists we say that coh-amp $E=\infty$.

Trivially coh-amp $E^{\bullet}$ is the minimal length of a bounded complex quasiisomorphic to $E^{\bullet}$, if any exist, and infinity, if none do.

Definition 2. Let $R$ be a ring or a sheaf of rings and $E^{\bullet}$ be a cochain complex of objects of Mod-R. Define its Tor-amplitude, denoted by Tor-amp $E_{R} E^{\bullet}$, to be the length of the minimal interval in $\mathbb{Z}$ containing the set

$$
\left\{i \in \mathbb{Z} \mid \exists A \in \operatorname{Mod}-R \text { such that } \operatorname{Tor}_{R}^{i}\left(E^{\bullet}, A\right) \neq 0\right\} .
$$

If no such interval exists we say that Tor-amp ${ }_{R} E=\infty$.

Def. 2 can be seen to be equivalent to [Kuz05], Def. 2.20.

Let now $X$ be any scheme. It follows from [Har66], Prop 4.2, that an object of $D^{b}(\operatorname{Mod}-X)$ has finite Tor-amplitude if and only if it is of finite Tordimension, i.e. quasi-isomorphic to a bounded complex of flat sheaves.

Lemma 3. Let $X$ be a locally noetherian scheme and $E \in D_{\text {coh }}^{b}(X)$ an object of finite Tor-dimension. Denote by $l$ the length of the shortest complex of flat sheaves quasi-isomorphic to $E$, and by $k$ the length of the smallest interval in $\mathbb{Z}$ containing the set

$$
\left\{i \in \mathbb{Z} \mid \exists x \in X \text { such that } \mathbf{L}^{i} \iota_{x}^{*}(E) \neq 0\right\} .
$$

Then $l=$ Tor-amp $\operatorname{O}_{X} E=k$.

Proof. Implications $l \geq$ Tor-amp $_{\mathcal{O}_{X}} E$ and Tor-amp $\mathcal{O}_{X} E \geq k$ are trivial. We claim that $k \geq l$. Let $n, k \in \mathbb{Z}$ be such that the interval $[-n-k,-n]$ contains the set (2.8). Then (2.3) and (2.4) of Proposition 1 show that $\mathcal{H}^{i}(E)=0$ unless $i \in[n, n+k]$. Since resolutions by flat modules exist on $X$, there exists a complex $F^{\bullet}$ of flat sheaves quasi-isomorphic to $E$ and with $F_{i}=0$ for all $i>n+k$. We claim that we can truncate $F^{\bullet}$ at degree $n$ and keep it flat, i.e. that the sheaf $F^{n} / \operatorname{Im} F^{n-1}$ is flat. But as $\mathcal{H}^{i}\left(F^{\bullet}\right)=0$ for $i<n$, the complex

$$
\cdots \rightarrow F^{n-2} \rightarrow F^{n-1} \rightarrow F^{n} \rightarrow 0 \rightarrow \ldots
$$


is a flat resolution of $F^{n} / \operatorname{Im} F^{n-1}$. Hence $\mathbf{L}^{1} \iota_{x}^{*}\left(F^{n} / \operatorname{Im} F^{n-1}\right)=\mathbf{L}^{-n+1} \iota_{x}^{*}(E)$ and so vanishes for all $x \in X$ by assumption. Thus we obtain a length $k$ complex of flat-sheaves quasi-isomorphic to $E$, i.e. $k \geq l$.

Whenever $X$ is a quasi-projective scheme, or any other scheme where there exist resolutions by locally-free sheaves, replacing the word 'flat' by the word 'locally-free' throughout Lemma 3 and its proof shows that for any $E \in D_{\text {coh }}^{b}(X)$ its Tor-amplitude is the length of the shortest complex of locally-free sheaves quasi-isomorphic to $E$. In other words, Tor-amp $\mathcal{O}_{X} E$ is the homological dimension of $E$ introduced in [BM02]. The following can thus be compared to the inequality hom.dim. $E \geq \operatorname{codim} C$ of [BM02]:

Theorem 2. Let $X$ be a locally noetherian scheme and $E \in D_{\text {coh }}^{b}(X)$ an object of finite Tor-dimension. Then

$$
\text { Tor-amp } \mathcal{O}_{X} E \geq \operatorname{codim} \operatorname{Supp} E+\text { coh-amp } E
$$

and for any irreducible component $C$ of Supp $E$ we have

$$
\text { Tor-amp } \mathcal{O}_{C} E_{C}=\operatorname{codim} C+\operatorname{coh}-\operatorname{amp}_{\mathcal{O}_{C}} E_{C} \text {. }
$$

Remark: To see that the inequality (2.9) can be strict, consider $X=\mathbb{A}^{1}$ and $E=\mathcal{O}_{X} \oplus \mathcal{O}_{x}$ for some closed point $x \in X$.

Proof. Denote by $\mathcal{H}^{i}$ the $i$ th cohomology sheaf of $E$. Set

$$
\begin{array}{rr}
n=\inf _{x \in \text { supp } E}\left\{i \in \mathbb{Z} \mid x \in \operatorname{Supp} \mathcal{H}^{i}\right\} & m=\sup _{x \in \text { supp }}\left\{i \in \mathbb{Z} \mid x \in \operatorname{Supp} \mathcal{H}^{i}\right\} \\
l=\inf _{x \in \text { supp } E}\left\{i \in \mathbb{Z} \mid \mathbf{L}^{i} \iota_{x}^{*} E \neq 0\right\} & h=\sup _{x \in \text { supp } E}\left\{i \in \mathbb{Z} \mid \mathbf{L}^{i} \iota_{x}^{*} E \neq 0\right\}
\end{array}
$$

and observe that $m-n=$ coh-amp $E$ and $h-l=$ Tor-amp $_{\mathcal{O}_{X}} E$ (Lemma 3).

By (2.3) of Proposition 1 we have

$$
-m=l .
$$

Let $D$ be any irreducible component of $\operatorname{Supp} \mathcal{H}^{n}$. We then have

$$
\operatorname{codim} \operatorname{Supp} E-n \leq \operatorname{codim} D-n=\sup \left\{i \in \mathbb{Z} \mid \mathbf{L}^{i} \iota_{x_{D}}^{*} E \neq 0\right\} \leq h
$$

with the middle equality due to (2.4) of Proposition 1 applied to $D$. Subtracting (2.11) from (2.12) we obtain $(m-n)+\operatorname{codim} \operatorname{Supp} E \leq(h-l)$. This shows (2.9).

To obtain (2.10) we observe that on Spec $\mathcal{O}_{C}$ the support of the localisation $E_{C}$ consists of a single point $x_{C}$. Therefore applying the above argument to $X^{\prime}=\operatorname{Spec} \mathcal{O}_{C}$ and $E^{\prime}=E_{C}$ we have $D=x_{C}=\operatorname{Supp} E^{\prime}$ which makes both the inequalities in (2.12) into equalities. 


\section{Derived McKay correspondence}

Given a scheme $S$ denote by $D_{q c}(S)$ (resp. $D(S)$ ) the full subcategory of the derived category of $\mathcal{O}_{S^{-}}$Mod consisting of complexes with quasi-coherent (resp. bounded and coherent) cohomology. For $S$ a scheme of finite type over $\mathbb{C}$ and $H$ a finite group acting on $S$ on the left by automorphisms an $H$-sheaf is a sheaf $\mathcal{E}$ of $\mathcal{O}_{S}$-modules equipped with a lift of the $H$-action to $\mathcal{E}$. For the technical details see [BKR01], Section 4. Denote by $\mathcal{O}_{S^{-}} \operatorname{Mod}^{H}\left(\right.$ resp. $\left.\mathrm{QCoh}^{H} S, \mathrm{Coh}^{H} S\right)$ the abelian category of $H$-sheaves (resp. quasi-coherent, coherent $H$-sheaves) on $S$ and by $D_{q c}^{H}(S)$ (resp. $D^{H}(S)$ ) the full subcategory of the derived category of $\mathcal{O}_{S}$ - Mod $^{H}$ consisting of complexes with quasi-coherent (resp. bounded and coherent) cohomology.

\subsection{Integral transforms}

Let $N$ and $M$ be schemes of finite type over $\mathbb{C}$. Denote by $\pi_{N}$ and $\pi_{M}$ the projections $N \times M \rightarrow N$ and $N \times M \rightarrow M$.

Definition 3. Let $E$ be an object of $D_{q c}(N \times M)$ of finite Tor-dimension. An integral transform $\Phi_{E}$ is a functor $D_{q c}(N) \rightarrow D_{q c}(M)$ defined by

$$
\Phi_{E}(-)=\mathbf{R} \pi_{M *}\left(E \stackrel{\mathbf{L}}{\otimes} \pi_{N}^{*}(-)\right) .
$$

The object $E$ is called the kernel of the transform. If $\Phi_{E}$ is an equivalence of categories it is further called a Fourier-Mukai transform.

If a group $G$ acts on $N$ and $M$ then, for any $E \in D_{q c}^{G}(N \times M)$ of finite Tordimension, (3.1) defines an integral transform $D_{q c}^{G}(N) \rightarrow D_{q c}^{G}(M)$. If the group action on $N$ is trivial denote by $\left(-\otimes \rho_{0}\right)$ the functor $D_{q c}(N) \rightarrow D_{q c}^{G}(N)$ which gives a sheaf the trivial $G$-equivariant structure. It is exact and has an exact left and right adjoint $(-)^{G}$, the functor of taking the $G$-invariant part ([BKR01], Section 4.2). We also use the terms integral and Fourier-Mukai transform for the functors $D_{q c}(N) \rightarrow D_{q c}^{G}(M)$ of the form $\Phi_{E}\left(-\otimes \rho_{0}\right)$ where $\Phi_{E}$ is some integral transform $D_{q c}^{G}(N) \rightarrow D_{q c}^{G}(M)$.

When $N$ and $M$ are smooth and proper varieties it is well known that $\Phi_{E}$ has a left adjoint $\Phi_{E^{\vee} \otimes \pi_{M}^{*}\left(\omega_{M}\right)[\operatorname{dim} M]}$ ([BO95], Lemma 1.2). The lemma below allows to generalise this to certain integral transforms between non-proper schemes. We use methods of Verdier-Deligne as per the exposition in [Del66] to which we refer the reader for all the necessary definitions.

Lemma 4. Let $N$ and $M$ be separable schemes of finite type over $\mathbb{C}$ with $M$ smooth of dimension $n$. Let $E \in D(N \times M)$ be of finite homological dimension with $\operatorname{Supp}(E)$ proper over $N$. Then the functor

$$
\pi_{N}^{*}(-) \otimes \stackrel{\mathbf{L}}{\otimes}: \quad D(N) \rightarrow D(N \times M)
$$


has a left adjoint

$$
\mathbf{R} \pi_{N *}\left(-\stackrel{\mathbf{L}}{\otimes} E^{\vee} \otimes \pi_{M}^{*}\left(\omega_{M}\right)\right)[n]: \quad D(N \times M) \rightarrow D(N) .
$$

Proof. First we compactify $M$ : choose an open immersion $M \hookrightarrow \bar{M}$ with $\bar{M}$ smooth and proper [Nag]. Then $\pi_{N}$ decomposes as an open immersion $\iota: N \times$ $M \hookrightarrow N \times \bar{M}$ followed by the projection $\bar{\pi}_{N}: N \times \bar{M} \rightarrow N$. As $\bar{\pi}_{N}$ is smooth and proper Grothendieck-Serre duality for smooth and proper morphisms (e.g. [Har66], VII4.3) implies that $\bar{\pi}_{N}^{*}: D(N) \rightarrow D(N \times \bar{M})$ has a left adjoint

$$
\mathbf{R} \bar{\pi}_{N *}(-) \otimes \bar{\pi}_{M}^{*} \omega_{\bar{M}}[n]
$$

where $\bar{\pi}_{M}: N \times \bar{M} \rightarrow \bar{M}$ is the projection onto the second component.

By the duality for open immersions ([Del66], Prop. 4) the left adjoint to the (exact) functor $\iota^{*}(-)$ exists as an (exact) functor $\iota$ from $\operatorname{Coh}(N \times M)$ to the category pro-Coh $(N \times \bar{M})$. For the definition of $\operatorname{pro}-\operatorname{Coh}(N \times \bar{M})$ and the generalities on pro-objects see [Del66], $\mathrm{n}^{\circ} 1$. The functor $\iota$ ! may be calculated as follows: given $\mathcal{A} \in \operatorname{Coh}(N \times M)$ take any $\overline{\mathcal{A}} \in \operatorname{Coh}(N \times \bar{M})$ which restricts to $\mathcal{A}$ on $N \times M$. Then

$$
\iota_{!}(\mathcal{A})=\underline{\lim } \operatorname{Hom}\left(\mathcal{I}^{n} \overline{\mathcal{A}},-\right)
$$

where $\mathcal{I}$ is the ideal sheaf defining the complement $N \times(\bar{M} \backslash M)$.

Finally, as $E$ is of finite homological dimension, the left adjoint of $(-) \stackrel{\mathbf{L}}{\otimes} E$ is $(-) \stackrel{\mathbf{L}}{\otimes} E^{\vee}$ where $E^{\vee}$ is $\mathbf{R} \operatorname{Hom}\left(E, \mathcal{O}_{N \times M}\right)$.

Therefore the left adjoint of $\pi_{N}^{*}(-) \stackrel{\mathbf{L}}{\otimes} E$ exists as the functor

$$
\mathbf{R} \bar{\pi}_{N *}\left(\iota !\left(-\stackrel{\mathbf{L}}{\otimes} E^{\vee}\right) \otimes \bar{\pi}_{M}^{*}\left(\omega_{M}\right)\right)[n]
$$

from pro $-D(N \times M)$ to pro $-D(N)$. To finish the proof it suffices now to show that $\iota_{!}\left(-\stackrel{\mathbf{L}}{\otimes} E^{\vee}\right)=\iota_{*}\left(-\stackrel{\mathbf{L}}{\otimes} E^{\vee}\right)$. Then applying the projection formula to $\iota_{*}(-\stackrel{\mathbf{L}}{\otimes}$ $\left.E^{\vee}\right) \otimes \bar{\pi}_{M}^{*}\left(\omega_{M}\right)$ in (3.4) and observing that $\iota \circ \bar{\pi}_{M}=\pi_{M}$ and $\iota \circ \bar{\pi}_{N}=\pi_{N}$ yields (3.2).

We have Id $=\iota^{*} \iota_{*}$ on $\mathrm{QCoh}(N \times M)$ ([GD60], Prop. 9.4.2). It induces by the adjunction of [De166], Prop. 4 natural transformations $Y: \iota ! \rightarrow \iota_{*}$ of functors $\operatorname{Coh}(N \times M) \rightarrow \operatorname{pro}-\mathrm{QCoh}(N \times \bar{M})$ and $\Upsilon^{\prime}: \quad \iota !\left(-\stackrel{\mathbf{L}}{\otimes} E^{\vee}\right) \rightarrow$ $\iota_{*}\left(-\stackrel{\mathrm{L}}{\otimes} E^{\vee}\right)$ of functors $D(N \times M) \rightarrow$ pro $-D(N \times \bar{M})$. By [Del66], Prop. 3 and the exactness of $\iota$ ! and $\iota_{*}$, to show $\Upsilon^{\prime}$ to be an isomorphism of functors it suffices to show that $\Upsilon$ is an isomorphism on the cohomology sheaves of 
$-\stackrel{\mathbf{L}}{\otimes} E^{\vee}$. The support of these is proper over $N$ by the assumption on $E$. For any $\mathcal{A} \in \operatorname{Coh}(N \times M)$ we have

$$
\operatorname{Hom}\left(\iota_{!}(\mathcal{A}), \iota_{*}(\mathcal{A})\right)=\lim _{\longrightarrow} \operatorname{Hom}_{N \times \bar{M}}\left(\mathcal{I}^{k} \overline{\mathcal{A}}, \iota_{*}(\mathcal{A})\right)
$$

using the notation of (3.3). From the construction of the adjunction in [Del66], Prop. 4 it is immediate that $\Upsilon(\mathcal{A})$ is the unique element of RHS of (3.5) which restricts to $N \times M$ as Id $\in \operatorname{Hom}_{N \times M}(\mathcal{A}, \mathcal{A})$. If $\operatorname{Supp}(\mathcal{A})$ is proper over $N$, we can take $\overline{\mathcal{A}}=\iota_{*} \mathcal{A}$ in (3.3). Moreover, $\mathcal{I}^{k} \iota_{*}(\mathcal{A})=\iota_{*}(\mathcal{A})$ for all $k$. Therefore (3.3) yields $\iota_{!}(\mathcal{A})=\iota_{*}(\mathcal{A})$ and moreover the RHS of (3.5) is just $\operatorname{Hom}\left(\iota_{*} \mathcal{A}, \iota_{*} \mathcal{A}\right)$. It is then clear that $\Upsilon(\mathcal{A})=\mathrm{Id}$, as required.

\subsection{G-constellations and gnat-families}

Definition 4. Let $G$ be a finite subgroup of $\mathrm{GL}_{n}(\mathbb{C})$. A $G$-constellation is a coherent $G$-sheaf $\mathcal{V}$ on $\mathbb{C}^{n}$ whose global sections $\Gamma(\mathcal{V})$ have the $G$-representation structure of the regular representation $V_{\text {reg. }}$.

Two $G$-constellations $\mathcal{V}, \mathcal{W}$ are orthogonal in degree $k$ if $G$-Ext $\mathbb{\mathbb { C }}^{n}(\mathcal{V}, \mathcal{W})=$ $G-\operatorname{Ext}_{\mathbb{C}^{n}}^{k}(\mathcal{W}, \mathcal{V})=0$

Let now $Y$ be a scheme of finite type over $\mathbb{C}$. We endow $Y$ with the trivial $G$-action, thus we can speak of $G$-sheaves on $Y$ and on $Y \times \mathbb{C}^{n}$.

Definition 5. A gnat-family on $Y$ (short for $G$-natural or geometrically natural) is an object $\mathcal{F}$ of $\operatorname{Coh}^{G}\left(Y \times \mathbb{C}^{n}\right)$, flat over $Y$, such that for every closed $y \in Y$ the fiber $\mathcal{F}_{\mid y}$ is a $G$-constellation supported on a single $G$-orbit. The HilbertChow map $\pi_{\mathcal{F}}$ of $\mathcal{F}$ is the map $Y \rightarrow \mathbb{C}^{n} / G$ defined by $y \mapsto \operatorname{Supp}_{\mathbb{C}^{n}} \mathcal{F}_{\mid y}$. A gnat-family on a fixed morphism $Y \stackrel{\pi}{\rightarrow} \mathbb{C}^{n} / G$ is a gnat-family on $Y$ whose Hilbert-Chow map coincides with $\pi$.

Two subsets $C$ and $C^{\prime}$ of $Y$ are orthogonal in degree $k$ in $\mathcal{F}$ if for every $y \in C$ and $y^{\prime} \in C^{\prime}$ the fibers $\mathcal{F}_{\mid y}$ and $\mathcal{F}_{\mid y^{\prime}}$ are orthogonal in degree $k$. The family $\mathcal{F}$ is orthogonal in degree $k$ if $Y$ is orthogonal to $Y$ in degree $k$ in $\mathcal{F}$.

Proposition 2. For any gnat-family $\mathcal{F}$ its Hilbert-Chow map $\pi_{\mathcal{F}}$ is a morphism.

Proof. Denote by $R$ the ring $\mathbb{C}\left[x_{1}, \ldots, x_{n}\right]$. For any $G$-constellation $\mathcal{V}$, the action of $R$ on $H^{0}(\mathcal{V})$ restricts to the action of $R^{G}$ on $H^{0}(\mathcal{V})^{G}$. Clearly

$$
\left(\operatorname{Ann}_{R} H^{0}(\mathcal{V})\right)^{G} \subseteq \operatorname{Ann}_{R^{G}} H^{0}(\mathcal{V})^{G} .
$$

The LHS of (3.6) is the image of $\operatorname{Supp}_{\mathbb{C}^{n}} \mathcal{V}$ in $\mathbb{C}^{n} / G$. If this support is a single $G$-orbit, then $\left(\operatorname{Ann}_{R} H^{0}(\mathcal{V})\right)^{G}$ is maximal in $R^{G}$ and (3.6) is an equality. Therefore it suffices to construct a morphism $Y \rightarrow \mathbb{C}^{n} / G$ which sends each $y \in Y$ to $\operatorname{Ann}_{R^{G}} H^{0}\left(\mathcal{F}_{\mid y}\right)^{G}$. We construct it thus: the invariant part of $\pi_{Y *}(\mathcal{F})$ 
is a line bundle on $Y$, which has a $R^{G}$-module structure induced from $\mathcal{F}$. This structure defines a homomorphism $R^{G} \rightarrow \mathcal{O}_{Y}$. The corresponding morphism $Y \rightarrow \mathbb{C}^{n} / G$ is easily seen to send each $y \in Y$ to $\operatorname{Ann}_{R^{G}} H^{0}\left(\mathcal{F}_{\mid y}\right)^{G}$.

Lemma 5. If $\mathcal{F}$ is a gnat-family on $Y$ and $\pi_{\mathcal{F}}: Y \rightarrow \mathbb{C}^{n} / G$ is proper, then $\mathcal{F}$ is of finite homological dimension in $D^{G}\left(Y \times \mathbb{C}^{n}\right)$ and the integral transform $\Phi_{\mathcal{F}}: D_{q c}^{G}(Y) \rightarrow D_{q c}^{G}\left(\mathbb{C}^{n}\right)$ restricts to $D^{G}(Y) \rightarrow D^{G}\left(\mathbb{C}^{n}\right)$.

Proof. Let $\iota$ be the open immersion $Y \times \mathbb{C}^{n} \rightarrow Y \times \mathbb{P}^{n}$. As $\operatorname{Supp} \mathcal{F}$ is proper over $Y, \iota_{*} \mathcal{F}$ is coherent. Quite generally, given any coherent sheaf $\mathcal{A}$ on $Y \times \mathbb{P}^{n}$ flat over $Y$, consider the adjunction co-unit $\xi: \pi_{Y}^{*} \pi_{Y *} \mathcal{A} \rightarrow \mathcal{A}$. As $\pi_{Y}$ is proper and $\mathcal{A}$ is flat over $Y, \pi_{Y}^{*} \pi_{Y *} \mathcal{A}$ is lffr (locally free of finite rank). Twisting by some power of $\pi_{\mathbb{P}^{n}}^{*} \mathcal{O}(1)$ we can make $\xi$ surjective. But then $\operatorname{ker} \xi$ is again coherent and flat over $Y$. We set initially $\mathcal{A}=\iota_{*} \mathcal{F}$ and repeat this construction until ker $\xi$ becomes lffr. This has to happen eventually as $\iota_{*} \mathcal{F}$ is flat over $Y$ and $\mathbb{P}^{n}$ is smooth. Thus we obtain an lffr resolution of $\iota_{*} \mathcal{F}$ of finite length. Restricting it to $Y \times \mathbb{C}^{n}$ demonstrates the first claim.

For the second claim: since $\pi_{Y}$ is flat, the pullback $\pi_{Y}^{*}\left(-\otimes \rho_{0}\right)$ is exact and takes $D(Y)$ to $D^{G}\left(Y \times \mathbb{C}^{n}\right)$. Since $\mathcal{F}$ is of finite homological dimension, $\mathcal{F} \stackrel{\mathbf{L}}{\otimes}-$ takes $D^{G}\left(Y \times \mathbb{C}^{n}\right)$ to $D^{G}\left(Y \times \mathbb{C}^{n}\right)$. Moreover the image $\operatorname{Im}(\mathcal{F} \stackrel{\mathbf{L}}{\otimes}-)$ lies in the full subcategory of $D^{G}\left(Y \times \mathbb{C}^{n}\right)$ consisting of the objects with support in $\operatorname{Supp} \mathcal{F}$. Finally, $\pi_{\mathcal{F}}$ being proper implies that $\operatorname{Supp} \mathcal{F}$ is proper over $\mathbb{C}^{n}$, hence $\mathbf{R} \pi_{\mathbb{C}^{n} *}$ takes $\operatorname{Im}(\mathcal{F} \stackrel{\mathbf{L}}{\otimes}-)$ to $D^{G}\left(\mathbb{C}^{n}\right)$ ([GD61], Corollaire 3.2.4).

The following demonstrates a certain relevance of gnat-families:

Proposition 3. Let $G$ be a finite subgroup of $\mathrm{SL}_{n}(\mathbb{C}), Y$ a variety and $E \in$ $D^{G}\left(Y \times \mathbb{C}^{n}\right)$ an object such that $\Phi_{E}\left(-\otimes \rho_{0}\right)$ is an equivalence $D(Y) \stackrel{\sim}{\longrightarrow}$ $D^{G}\left(\mathbb{C}^{n}\right)$ which sends point sheaves on $Y$ to pure sheaves. Then $E$ is a gnatfamily over $Y$ and its Hilbert-Chow map $\pi_{E}$ is a crepant resolution of $\mathbb{C}^{n} / G$. Moreover

$$
G-\operatorname{Ext}^{i}\left(E_{\mid y_{1}}, E_{\mid y_{2}}\right)= \begin{cases}\mathbb{C} & \text { if } y_{1}=y_{2}, i=0 \\ 0 & \text { if } y_{1} \neq y_{2}\end{cases}
$$

and for any $y \in Y$ the (Kodaira-Spencer) map $\operatorname{Ext}^{1}\left(\mathcal{O}_{y}, \mathcal{O}_{y}\right) \rightarrow G$ - $\operatorname{Ext}^{1}\left(E_{\mid y}, E_{\mid y}\right)$ is an isomorphism.

Proof. By [Huy06], Example 5.1(vi), $E_{\mid y}=\Phi_{E}\left(\mathcal{O}_{y} \otimes \rho_{0}\right)$, whence the assertion (3.7) and the Kodaira-Spencer maps being isomorphisms. By [Bri99], Lemma 4.3, it follows that $E$ is a pure sheaf flat over $Y$. Then by Lemma 4 the inverse of $\Phi_{E}\left(-\otimes \rho_{0}\right)$ is $\Phi_{E^{\vee}[n]}(-)^{G}$. It maps $\mathcal{O}_{\mathbb{C}^{n}}$ to $\left(\pi_{Y *} E^{\vee}[n]\right)^{G}$, so the cohomology sheaves of $\left(\pi_{Y *} E^{\vee}[n]\right)^{G}$ are coherent $\mathcal{O}_{Y}$-modules. Since $\pi_{Y *}$ is affine, the 
support of $E^{\vee}[n]$ is finite over $Y$. As $\operatorname{Supp}\left(E^{\vee}[n]\right)=\operatorname{Supp} E$, we conclude that for each $y \in Y$ the support of $E_{\mid y}$ is a finite union of $G$-orbits. The simplicity of $E_{\mid y}$ further implies that it has to be a single $G$-orbit. To show that $\Gamma\left(E_{\mid y}\right)$ has $G$-representation structure of $V_{\text {reg }}$ it suffices, by flatness of $E$, to show it for any single $y \in Y$. As the set $\left\{E_{\mid y}\right\}_{y \in Y}$ is an image of a spanning class of $D(Y)$ under $\Phi\left(-\otimes \rho_{0}\right)$, it is a spanning class for $D^{G}\left(\mathbb{C}^{n}\right)$. Hence for every $G$-orbit $Z$ in $\mathbb{C}^{n}$ there exists $y \in Y$ such that $E_{\mid y}$ is supported at $Z$. Choose $Z$ to be any free orbit. The only simple $G$-sheaf supported on a free orbit is its structure sheaf, therefore $\Gamma\left(E_{\mid y}\right) \simeq V_{\text {reg. }}$. We conclude that $E$ is a gnat-family and that $\pi_{E}$ is surjective and an isomorphism outside the singularities of $\mathbb{C}^{n} / G$. By [Rob98], Theorem 6.2.2 and [BKR01], Lemma 3.1, $Y$ is smooth and $\pi_{E}$ is crepant. It remains to show that $\pi_{E}$ is proper, which is equivalent to $\operatorname{Supp}_{Y \times \mathbb{C}^{n}} E$ being proper over $\mathbb{C}^{n}$ and that follows, e.g., from $\pi_{\mathbb{C}^{n} *} E$ having to be coherent, as it is a cohomology sheaf of the complex $\Phi_{E}\left(\mathcal{O}_{Y} \otimes \rho_{0}\right)$.

\subsection{Main results}

We now give the proof of Theorem 1. Its general framework follows those of [BO95], Theorem 1.1 and of [BKR01], Theorem 1.1. We note two principal differences: [BO95] works with smooth varieties, while we assume $Y$ to be a not necessarily smooth scheme (whence the content of Section 2); [BKR01] adopts a two-step strategy to establish the left adjoint of $\Phi_{\mathcal{F}}\left(-\otimes \rho_{0}\right)$, whereas our Lemma 4 achieves this directly.

Proof (Proof of Theorem 1).

We divide the proof into five steps:

Step 1: We claim that $\Phi_{\mathcal{F}}\left(-\otimes \rho_{0}\right)$ has a left adjoint $\left(\Psi_{\mathcal{F}}\right)^{G}$, where $\Psi_{\mathcal{F}}$ is a certain integral transform $D^{G}\left(\mathbb{C}^{n}\right) \rightarrow D^{G}(Y)$.

Recall that $\Phi_{\mathcal{F}}=\mathbf{R} \pi_{\mathbb{C}^{n} *}\left(\mathcal{F} \stackrel{\mathbf{L}}{\otimes} \pi_{Y}^{*}(-)\right)$. The issue here is the left adjoint of $\pi_{Y}^{*}(-)$ as $\pi_{Y}$, though smooth, is manifestly non-proper. But the support of $\mathcal{F}$ is proper, so by Lemma 4 the functor $\mathbf{R} \pi_{Y *}\left(-\stackrel{\mathbf{L}}{\otimes} \mathcal{F}^{\vee}[n]\right)$ is the left adjoint to $\pi_{Y}^{*}(-) \stackrel{\mathbf{L}}{\otimes} \mathcal{F}$. The claim now follows, for $\pi_{\mathbb{C}^{n}}^{*}$ is the left adjoint to $\mathbf{R} \pi_{\mathbb{C}^{n} *}$ and $(-)^{G}$ is the left (and right) adjoint of $-\otimes \rho_{0}$.

Step 2: We claim that the composition $\left(\Psi_{\mathcal{F}}\right)^{G} \circ \Phi_{\mathcal{F}}\left(-\otimes \rho_{0}\right)$ is an integral transform $\Phi_{Q}$ for some $Q \in D(Y \times Y)$ and that for any closed point $\left(y_{1}, y_{2}\right)$ in $Y \times Y$ and any $k \in \mathbb{Z}$ we have

$$
\mathbf{L}^{k} \iota_{y_{1}, y_{2}}^{*} Q=G-\operatorname{Ext}^{k}\left(\mathcal{F}_{\mid y_{1}}, \mathcal{F}_{\mid y_{2}}\right)^{\vee} .
$$

The first assertion is a standard result due to Mukai in [Muk81], Proposition 1.3. The second assertion follows from the formula (5) of [BKR01], Sec. 6, Step 2 by the adjunction of $\mathbf{L} \iota_{y_{1}, y_{2}}^{*}$ and $\iota_{y_{1}, y_{2} *}$. 
Step 3: We claim that $Q$ is a pure sheaf and that its support lies within the diagonal $Y \stackrel{\Delta}{\longrightarrow} Y \times Y$.

First note that since $Y \times Y$ is of finite type over $\mathbb{C}$, it is certainly Jacobson (see [GD66], §10.3) and so any closed set of $Y \times Y$ is uniquely identified by its set of closed points. We implicitly use this property at several points of the argument below.

Recall the closed set $N_{k}$ of (1.2). As the support of any $G$-constellation is proper and as $\omega_{\mathbb{C}^{n}}=\mathcal{O}_{\mathbb{C}^{n}} \otimes \rho_{0}$ as a $G$-sheaf since $G \subseteq \mathrm{SL}_{n}(\mathbb{C})$, Serre duality applies to yield

$$
G-\operatorname{Ext}_{\mathbb{C}^{n}}^{k}\left(\mathcal{F}_{\mid y_{1}}, \mathcal{F}_{\mid y_{2}}\right)=G-\operatorname{Ext}_{\mathbb{C}^{n}}^{n-k}\left(\mathcal{F}_{\mid y_{2}}, \mathcal{F}_{\mid y_{1}}\right)^{\vee}
$$

It follows that $\operatorname{codim} N_{k}=\operatorname{codim} N_{n-k}$ for all $k$.

Let $C$ be an irreducible component of $\operatorname{Supp} Q$. Denote by $y_{C}$ its generic point, by $\mathcal{O}_{C}$ the local ring of $y_{C}$ and by $Q_{C}$ the localisation of $Q$ to $\mathcal{O}_{C}$. For any $k$ denote by $M_{k}$ the set $\left\{y \in Y \times Y \mid \mathbf{L}^{k} \iota_{y}^{*} Q \neq 0\right\}$ and let $l$ and $m$ be the infimum and the supremum of the set $\left\{k \in \mathbb{Z} \mid y_{C} \in M_{k}\right\}$, hence Tor-amp $\mathcal{O}_{C} Q_{C}=m-l$ (Lemma 3). By (3.8) the closure of $M_{l} \backslash \Delta$ is $N_{l}$, so $y_{C} \in M_{l}$ implies $y_{C} \in \Delta$ or $y_{C} \in N_{l}$. Similarly for $N_{m}$. Thus either $y_{C} \in \Delta$ or $y_{C} \in N_{l} \cap N_{m}$. The latter would imply that

$$
\begin{aligned}
& \operatorname{codim} C \geq \operatorname{codim} N_{l} \geq n-2 l+1 \\
& \operatorname{codim} C \geq \operatorname{codim} N_{m}=\operatorname{codim} N_{n-m} \geq 2 m-n+1
\end{aligned}
$$

and therefore that $\operatorname{codim} C \geq m-l+1$. But then $\operatorname{codim} C$ would be strictly greater than Tor-amp $\mathcal{O}_{C} Q_{C}$, which contradicts Theorem 2. Thus $y_{C}$ lies within $\Delta$ and, since $Y$ is separated, so does all of $C$.

We have now shown that $\operatorname{Supp} Q \subseteq \Delta$, so codim $\operatorname{Supp} Q \geq n$. But as $\mathbb{C}^{n}$ is smooth and $n$-dimensional, (3.8) implies

$$
\mathbf{L}^{k} \iota_{y}^{*} Q=0 \quad \forall y \in Y, k \notin 0, \ldots, n
$$

so Tor-amp $Q \leq n$. By Theorem 2 Tor-amp $Q=n$ and coh-amp $Q=0$. Together with (3.9) this implies that $Q$ is a pure sheaf.

Step 4: We claim that $Q$ is the structure sheaf $\mathcal{O}_{\Delta}$ of the diagonal $\Delta$ and therefore $\Phi_{\mathcal{F}}\left(-\otimes \rho_{0}\right)$ is fully faithful.

The adjunction co-unit $\Phi_{Q} \rightarrow \operatorname{Id}_{D(Y)}$ induces a surjective $\mathcal{O}_{Y \times Y}$-module morphism $Q \stackrel{\epsilon}{\rightarrow} \mathcal{O}_{\Delta}$. Let $K$ be its kernel, we then have a short exact sequence

$$
0 \rightarrow K \rightarrow Q \stackrel{\epsilon}{\rightarrow} \mathcal{O}_{\Delta} \rightarrow 0 .
$$

Choosing some closed point $(y, y) \in \Delta$ and applying functor $\mathbf{L} \iota_{y, y}^{*}(-)$ to (3.10) we obtain a long exact sequence of $\mathbb{C}$-modules

$\cdots \rightarrow G-\operatorname{Ext}_{\mathbb{C}^{n}}^{1}\left(\mathcal{F}_{\mid y}, \mathcal{F}_{\mid y}\right)^{*} \stackrel{\alpha_{y}}{\longrightarrow} \Omega_{Y, y}^{1} \rightarrow K_{y, y} \rightarrow G-\operatorname{End}_{\mathbb{C}^{n}}\left(\mathcal{F}_{\mid y}\right)^{*} \stackrel{\epsilon_{y}}{\longrightarrow} \mathbb{C} \rightarrow 0 \rightarrow \ldots$ 
The map $\epsilon_{y}$ is surjective due to any $G$-constellation having automorphisms consisting of scalar multiplication. It is an isomorphism whenever $\mathcal{F}_{\mid y}$ is simple, i.e. when the scalar multiplication automorphisms are all we get. The map $\alpha_{y}$ is the dual of the Kodaira-Spencer map of $\mathcal{F}$ at $y \in Y$, which takes a tangent vector at $y$ to the infinitesimal deformation in that direction in the family $\mathcal{F}$. Hence for any $y \in Y$, such that $\mathcal{F}_{\mid y}$ is simple and such that the Kodaira-Spencer map of $\mathcal{F}$ is injective at $y$, the long exact sequence above shows that $\left.K\right|_{y, y}=0$.

Having proved that $\operatorname{Supp} Q \subseteq \Delta$ we have proved by (3.8) that any two $G$-constellations in $\mathcal{F}$ are orthogonal. Denoting by $q$ the quotient map $\mathbb{C}^{n} \rightarrow$ $\mathbb{C}^{n} / G$ we claim that for any closed point $x \in \mathbb{C}^{n} / G$, such that $q^{-1}(x)$ is a free orbit of $G$, the fiber $\pi_{\mathcal{F}}^{-1}(x)$ consists of at most a single point. This is because, by definition of $\pi_{\mathcal{F}}$, all the $G$-constellations parametrised by $\pi_{\mathcal{F}}^{-1}(x)$ are supported on $q^{-1}(x)$ - and any two $G$-constellations supported at the same free orbit are easily seen to be isomorphic. Thus $\pi_{\mathcal{F}}$ is an isomorphism on the smooth locus $X_{0}$ of $\mathbb{C}^{n} / G$. By [Log06], Proposition 1.5 the family $\mathcal{F}$ on $X_{0}$ (identified with an open subset of $Y$ via $\pi_{\mathcal{F}}$ ) is locally isomorphic to the canonical $G$-cluster family $\left.q_{*} \mathcal{O}_{\mathbb{C}^{n}}\right|_{X_{0}}$. As any $G$-cluster is simple and as the KodairaSpencer map of $\left.q_{*} \mathcal{O}_{\mathbb{C}^{n}}\right|_{X_{0}}$ is trivially injective $\left.K\right|_{y, y}=0$ for any $y \in X_{0}$. Therefore $\operatorname{codim}_{Y \times Y} \operatorname{Supp} K \geq n+1$, as $X_{0}$ is open in $\Delta$.

On the other hand, since Tor-amp $Q=$ Tor-amp $\mathcal{O}_{\Delta}=n$, the short exact sequence (3.10) implies that Tor-amp $K \leq n$. As that is smaller than the codimension of its support, $K=0$ by Theorem 2 . Thus $Q \simeq \mathcal{O}_{\Delta}$, the adjunction co-unit is an isomorphism and $\Phi_{\mathcal{F}}\left(-\otimes \rho_{0}\right)$ is fully faithful.

Step 5: We claim that $\Phi_{\mathcal{F}}\left(-\otimes \rho_{0}\right)$ is an equivalence of categories.

As $D(Y)$ is fully faithfully embedded in $D^{G}\left(\mathbb{C}^{n}\right)$ the trivial Serre functor of the latter induces a trivial Serre functor on the former. Therefore the left adjoint to $\Phi_{\mathcal{F}}\left(-\otimes \rho_{0}\right)$ is also its right adjoint. Then $\Phi_{\mathcal{F}}\left(-\otimes \rho_{0}\right)$ is an equivalence of categories by [Bri99], Theorem 3.3.

\section{Proof (Proof of Corollary 1).}

It suffices to demonstrate that $\mathcal{F}$ satisfies the condition of Theorem 1 . Thus we have to show that codim $N_{0} \geq 4$ and $\operatorname{codim} N_{1} \geq 2$. But, as seen in the proof of Theorem 1, $N_{k}$ lies within the fibre product $Y \times_{\mathbb{C}^{3} / G} Y$ for all $k$. As $\pi_{\mathcal{F}}$ is birational its fibres are at most divisors and so the codimension of $Y \times{ }_{\mathbb{C}^{3} / G} Y$ is at least 2 .

It remains to show that $N_{0} \geq 4$. The assumptions of the Corollary ensure that $N_{0}$ is contained in the union of all sets of form $\left(E_{i} \cap E_{j}\right) \times\left(E_{k} \cap E_{l}\right)$ or $E_{i} \times\left(E_{i} \cap E_{j} \cap E_{k}\right)$, and the codimension of each of these sets is 4 .

\section{Orthogonality in degree zero}

Throughout this section we denote by $G$ a finite abelian subgroup of $\mathrm{SL}_{n}(\mathbb{C})$, by $Y$ a smooth scheme of finite type over $\mathbb{C}$ and by $\mathcal{F}$ a gnat-family on $Y$. We 
assume that the Hilbert-Chow morphism $\pi_{\mathcal{F}}$ associated to $\mathcal{F}$ is birational and proper. The main purpose of this section is to show how, given any pair of closed points of $Y$, one checks whether the corresponding pair of $G$-constellations are orthogonal in degree 0.

We denote by $V_{\text {giv }}$ the representation of $G$ given by its inclusion into $\mathrm{SL}_{n}(\mathbb{C})$. The (left) action of $G$ on $V_{\text {giv }}$ induces a right action of $G$ on $V_{\text {giv }}{ }^{\vee}$ which we make into a left action by setting:

$$
g \cdot f(v)=f\left(g^{-1} \cdot v\right) \quad \text { for all } v \in V_{\text {giv }}, f \in V_{\text {giv }} \vee, g \in G .
$$

We denote by $x_{1}, \ldots, x_{n}$ the common eigenvectors of the action of $G$ on $V_{\text {giv }}{ }^{\vee}$. We denote by $R$ the symmetric algebra $S\left(V_{\text {giv }}{ }^{\vee}\right)$ with the induced left action of $G$. Then $R=\mathbb{C}\left[x_{1}, \ldots, x_{n}\right]$ and as an affine $G$-scheme $\mathbb{C}^{n}$ is Spec $R$. We denote by $G^{\vee}$ the character group $\operatorname{Hom}\left(G, \mathbb{C}^{*}\right)$ of $G$. A rational function $f \in$ $K\left(\mathbb{C}^{n}\right)$ is said to be $G$-homogeneous of weight $\chi \in G^{\vee}$ if we have $f(g \cdot v)=$ $\chi(g) f(v)$ for all $v \in \mathbb{C}^{n}$ where $f$ is defined. We denote by $\rho(f)$ the weight $\chi$ of such $f$. It follows from (4.1) that $G$ acts on $f$ by $\rho(f)^{-1}$.

From here on we employ freely the terminology and the results of [ $\log 06]$.

\subsection{The McKay quiver of $G$}

By a quiver we mean a vertex set $Q_{0}$, an arrow set $Q_{1}$ and a pair of maps $h: Q_{1} \rightarrow Q_{0}$ and $t: Q_{1} \rightarrow Q_{0}$ giving the head $h q \in Q_{0}$ and the tail $t q \in Q_{0}$ of each arrow $q \in Q_{1}$. By a representation of a quiver we mean a graded vector space $\bigoplus_{i \in Q_{0}} V_{i}$ and a collection of linear maps $\left\{\alpha_{q}: V_{t q} \rightarrow V_{h q}\right\}_{q \in Q_{1}}$.

Definition 6. The McKay quiver of $G$ is the quiver whose vertex set $Q_{0}$ are the irreducible representations $\rho$ of $G$ and whose arrow set $Q_{1}$ has $\operatorname{dim} \operatorname{Hom}_{G}\left(\rho_{i}, \rho_{j} \otimes\right.$ $\left.V_{\text {giv }}\right)$ arrows going from the vertex $\rho_{i}$ to the vertex $\rho_{j}$.

We have $V_{\text {giv }}{ }^{\vee}=\bigoplus \mathbb{C} x_{i}$, as $G$-representations. Denote by $U_{\chi}$ the 1-dimensional representation on which $G$ acts by $\chi \in G^{\vee}$. By Schur's lemma

$$
G-\operatorname{Hom}\left(U_{\chi_{i}} \otimes V_{\text {giv }}{ }^{\vee}, U_{\chi_{j}}\right)=\left\{\begin{array}{ll}
\mathbb{C} & \text { if } \chi_{j}=\chi_{i} \rho\left(x_{k}\right)^{-1} \quad k \in\{1, \ldots, n\} \\
0 & \text { otherwise }
\end{array} .\right.
$$

Thus each vertex $\chi$ of the McKay quiver of $G$ has $n$ arrows emerging from it and going to vertices $\chi \rho\left(x_{k}\right)^{-1}$ for $k=1, \ldots, n$. We denote the arrow from $\chi$ to $\chi \rho\left(x_{k}\right)^{-1}$ by $\left(\chi, x_{k}\right)$. Let now $A$ be a $G$-constellation viewed as an $R \rtimes G$ module ([Log06], Section 1.1) and let $\oplus A_{\chi}$ be its decomposition into irreducible representations of $G$. Then the $R \rtimes G$-module structure on $A$ defines a representation of the McKay quiver into the graded vector space $\oplus A_{\chi}$, where the map $\alpha_{\chi, x_{k}}$ is just the multiplication by $x_{k}$, i.e.

$$
\alpha_{\chi, x_{k}}: A_{\chi} \rightarrow A_{\chi \rho\left(x_{k}\right)^{-1}}, v \mapsto x_{k} \cdot v .
$$




\subsection{Degree 0 orthogonality of $G$-constellations}

Let $A$ and $A^{\prime}$ be two $G$-constellations and $\phi$ be an $R \rtimes G$-module morphism $A \rightarrow A^{\prime}$. Let $\bigoplus_{G^{\vee}} A_{\chi}$ and $\bigoplus_{G^{\vee}} A_{\chi}^{\prime}$ be decompositions of $A$ and $A^{\prime}$ into onedimensional representations of $G$. By $G$-equivariance $\phi$ decomposes into linear maps $\phi_{\chi}: A_{\chi} \rightarrow A_{\chi}^{\prime}$.

Let $\left\{\alpha_{q}\right\}$ and $\left\{\alpha_{q}^{\prime}\right\}$ be the corresponding representations of the McKay quiver into graded vector spaces $\oplus A_{\chi}$ and $\oplus A_{\chi}^{\prime}$, as per (4.2). Each $\alpha_{q}$ is a linear map between one-dimensional vector spaces $A_{t q}$ and $A_{h q}$ and so is either a zero-map or an isomorphism, similarly for the maps $\alpha_{q}^{\prime}$. So for each arrow of the McKay quiver we distinguish the following four possibilities:

Definition 7. Let $q$ be an arrow of McKay quiver of $G$. With the notation above we say that with respect to an ordered pair $\left(A, A^{\prime}\right)$ of $G$-constellations the arrow qis:

1. a type $[1,1]$ arrow, if both $\alpha_{q}$ and $\alpha_{q}^{\prime}$ are isomorphisms.

2. a type $[1,0]$ arrow, if $\alpha_{q}$ is an isomorphism and $\alpha_{q}^{\prime}$ is a zero map.

3. a type $[0,1]$ arrow, if $\alpha_{q}$ is a zero map and $\alpha_{q}^{\prime}$ is an isomorphism.

4. a type $[0,0]$ arrow, if both $\alpha_{q}$ and $\alpha_{q}^{\prime}$ are zero maps.

Proposition 4. Let $q$ and $\left(A, A^{\prime}\right)$ be as in Definition 7 and let $\phi$ be any $R \rtimes G$ module morphism $A \rightarrow A^{\prime}$. Then:

1. If $q$ is a $[1,0]$ arrow, then $A_{h q} \subseteq \operatorname{ker} \phi$.

2. If $q$ is a $[0,1]$ arrow, then $A_{t q} \subseteq \operatorname{ker} \phi$.

3. If $q$ is a $[1,1]$ arrow, $A_{t q}$ and $A_{h q}$ either both lie in ker $\phi$ or both don't.

Proof. Write $q=(\chi, i)$ where $\chi \in G^{\vee}$ and $i \in\{1, \ldots, n\}$. Recall that $\alpha_{q}$ is the map $A_{t q} \rightarrow A_{h q}$ corresponding to the action of $x_{i}$ on $A_{t q}$. Then $R$-equivariance of the morphism $\phi$ implies a commutative square

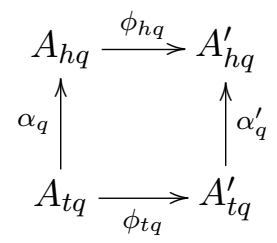

from which all three claims immediately follow.

Corollary 2. Let $\left(A, A^{\prime}\right)$ be an ordered pair of $G$-constellations. If every component of the McKay quiver path-connected by $[1,1]$-arrows has either a $[0,1]$ arrow emerging from it or a $[1,0]$-arrow entering it, then

$$
\operatorname{Hom}_{R \rtimes G}\left(A, A^{\prime}\right)=0 .
$$


If, also, every component has either a [0,1]-arrow entering it or a $[1,0]$-arrow emerging from it, then we further have

$$
\operatorname{Hom}_{R \rtimes G}\left(A^{\prime}, A\right)=0
$$

and therefore $A$ and $A^{\prime}$ are orthogonal in degree 0.

\subsection{Divisors of zeroes}

The Hilbert-Chow morphism $\pi_{\mathcal{F}}: Y \rightarrow \mathbb{C}^{n} / G$ is birational, thus it defines a notion of $G$-Cartier and $G$-Weil divisors on $Y$ ([Log06]), Section 2$)$. The family $\mathcal{F}$, in a sense of a sheaf of $\mathcal{O}_{Y} \otimes(R \rtimes G)$-modules on $Y$, can be written as $\bigoplus_{\chi \in G^{\vee}} \mathcal{L}\left(-D_{\chi}\right)$, where $D_{\chi}$ are $G$-Weil divisors. For any other such expression $\bigoplus \mathcal{L}\left(-D_{\chi}^{\prime}\right)$ of $\mathcal{F}$ there exist $f \in K(Y)$ such that $D_{\chi}^{\prime}=D_{\chi}+(f)$ for all $\chi \in G^{\vee}([\log 06]$, Section 3.1).

Definition 8. Let $q=\left(\chi, x_{k}\right)$ be an arrow in the McKay quiver of $G$. We define the divisor of zeroes $B_{q}$ of $q$ in $\mathcal{F}$ to be the Weil divisor

$$
D_{\chi^{-1}}+\left(x_{i}\right)-D_{\chi^{-1} \rho\left(x_{i}\right)} .
$$

Note that $B_{q}$ is always an ordinary, integral Weil divisor on $Y$.

Proposition 5. Let $\left(\chi, x_{k}\right)$ be an arrow in the McKay quiver of $G$ and $B_{\chi, x_{k}}$ be its divisor of zeroes in $\mathcal{F}$. Let $y$ be a closed point of $Y$ and $A$ be the $G$ constellation $\mathcal{F}_{\mid y}$. Then in the corresponding representation $\left\{\alpha_{q}\right\}_{q \in Q_{1}}$ of the McKay quiver the map $\alpha_{\chi, x_{k}}$ is a zero map if and only if $y \in B_{\chi, x_{k}}$.

Proof. The map $\alpha_{\chi, x_{k}}: A_{\chi} \rightarrow A_{\chi \rho\left(x_{k}\right)^{-1}}$ is the action of $x_{k}$ on $A_{\chi}$. This map is the restriction to the point $y$ of the global section $\beta$ of the $\mathcal{O}_{Y}$-module

$$
\operatorname{Hom}_{G, \mathcal{O}_{Y}}\left(\mathcal{O}_{Y} x_{k} \otimes \mathcal{F}_{\chi}, \mathcal{F}_{\chi \rho^{-1}\left(x_{k}\right)}\right)
$$

defined by $x_{k} \otimes s \mapsto x_{k} \cdot s$ for any section $s$ of the $\chi$-eigensheaf $\mathcal{F}_{\chi}$.

As $G$ acts on a monomial of weight $\chi$ by $\chi^{-1}$ the $\chi$-eigensheaf of $\mathcal{F}$ is $\mathcal{L}\left(-D_{\chi^{-1}}\right)$. Hence (4.4) is canonically isomorphic to the following sub- $\mathcal{O}_{Y^{-}}$ module of $K\left(\mathbb{C}^{n}\right)$ :

$$
\mathcal{L}\left(D_{\chi^{-1}}+\left(x_{k}\right)-D_{\chi^{-1} \rho\left(x_{k}\right)}\right)
$$

and the isomorphism maps $\beta$ to the global section $1 \in K\left(\mathbb{C}^{n}\right)$ of (4.5). Which vanishes precisely on the Weil divisor $B_{\chi, x_{k}}=D_{\chi^{-1}}+\left(x_{k}\right)-D_{\chi^{-1} \rho\left(x_{k}\right)}$.

Proposition 5 together with Corollary 2 show that the data of the divisors of zeroes of $\mathcal{F}$ is all that is necessary to determine whether any given pair of closed points of $Y$ are orthogonal in degree 0 in $\mathcal{F}$. 


\subsection{Direct transforms}

Let $Y^{\prime}$ and $Y^{\prime \prime}$ be two crepant resolutions of $\mathbb{C}^{n} / G$ isomorphic outside of a closed set of codimension $\geq 2$. E.g. the case $n=3$ where all crepant resolutions are related by a chain of flops ([Kol89]). We fix a birational isomorphism and use it to identify $Y^{\prime}$ and $Y^{\prime \prime}$ along the isomorphism locus $U$. Since the complement of $U$ is of codimension $\geq 2$ in $Y^{\prime}$ (resp. $Y^{\prime \prime}$ ) any line bundle or divisor on $U$ extends uniquely to a line bundle or a divisor on $Y^{\prime}$ (resp. $Y^{\prime \prime}$ ). The same is true of a family of $G$-constellations as for $G$ abelian any such family is a direct sum of line bundles. For any family $\mathcal{V}^{\prime}$ of $G$-constellations on $Y^{\prime}$ we define its direct transform $\mathcal{V}^{\prime \prime}$ to $Y^{\prime \prime}$ to be the unique extension to $Y^{\prime \prime}$ of the restriction of $\mathcal{V}^{\prime}$ to $U$. Observe that if $\mathcal{V}^{\prime}$ is of form $\bigoplus_{\chi} \mathcal{L}\left(-D_{\chi}^{\prime}\right)$ for some $G$-Weil divisors $D_{\chi}^{\prime}$ on $Y^{\prime}$ then $\mathcal{V}^{\prime \prime}$ is the family $\bigoplus \mathcal{L}\left(-D_{\chi}^{\prime \prime}\right)$ where each $D_{\chi}^{\prime \prime}$ is the direct transform of $D_{\chi}^{\prime}$.

If $\mathcal{F}$ can be shown to be a direct transform of some everywhere orthogonal in degree 0 family $\mathcal{F}^{\prime}$ on some $Y^{\prime}$, it greatly reduces the number of calculations necessary to determine the degree 0 non-orthogonality locus of $\mathcal{F}$. Let $U$ be as above. As $\mathcal{F}$ is the direct transform of $F^{\prime}$, the restriction of $\mathcal{F}$ to $U \subset Y$ is isomorphic to the restriction of $F^{\prime}$ to $U \subset Y^{\prime}$. So the calculations only have to be carried out for points in $Y \times Y \backslash U \times U$.

\subsection{Theta stability and gnat-families}

We recall basic facts about $\theta$-stability for $G$-constellations, cf. [CI04], Section 2.1. Let $\mathbb{Z}(G)=\bigoplus_{\chi \in G^{\vee}} \mathbb{Z} \chi$ be the representation ring of $G$ and set

$$
\Theta=\left\{\theta \in \operatorname{Hom}_{\mathbb{Z}}(\mathbb{Z}(G), \mathbb{Q}) \mid \theta\left(V_{\text {reg }}\right)=0\right\}
$$

For any $\theta \in \Theta$, a $G$-constellation $A$ is $\theta$-stable (resp. $\theta$-semistable) if for every sub- $R \rtimes G$-module $B$ of $A$ we have $\theta(B)>0$ (resp. $\theta(B) \geq 0$ ). We say that $\theta$ is generic if every $\theta$-semistable $G$-constellation is $\theta$-stable. This is equivalent to $\theta$ being non-zero on any proper subrepresentation of $V_{\text {reg }}$.

Let $\pi$ be any proper birational morphism $Y \rightarrow \mathbb{C}^{n} / G$. A gnat-family $\mathcal{V}$ on $Y \stackrel{\pi}{\rightarrow} \mathbb{C}^{n} / G$ is normalized if $\mathcal{V}^{G} \simeq \mathcal{O}_{Y}$. Such $\mathcal{V}$ can be written uniquely as $\bigoplus_{\chi \in G^{\vee}} \mathcal{L}\left(-D_{\chi}\right)$ for some $G$-Weil divisors $D_{\chi}$ with $D_{\chi_{0}}=0$ ([Log06], Cor. 3.5). Denote by $\mathfrak{E}$ the set of all prime Weil divisors on $Y$ whose image in $\mathbb{C}^{n} / G$ is either a point or a coordinate hyperplane $x_{i}^{|G|}=0$. As $G$ is abelian, any branch divisor of $\mathbb{C}^{n} \rightarrow \mathbb{C}^{n} / G$, if it exists, is one of the hyperplanes $x_{i}^{|G|}=0$. Hence, by [Log06], Prop. 3.14 and 3.15, each $D_{\chi}$ is of form $\sum_{E \in \mathfrak{E}} q_{\chi, E} E$. Denote by $U$ the open subset of $Y$ consisting of points lying on at most one divisor in $\mathfrak{E}$. 
Definition 9. Let $\theta$ be an element of $\Theta$. We define a map

$$
w_{\theta}: \quad\left\{\text { normalized gnat-families on } Y \stackrel{\pi}{\rightarrow} \mathbb{C}^{n} / G\right\} \rightarrow \mathbb{Q}
$$

by

$$
w_{\theta}(\mathcal{V})=\sum_{E \in \mathfrak{E}} \sum_{\chi \in G^{\vee}} \theta(\chi) q_{\chi, E}
$$

The domain of definition of $w_{\theta}$ is finite ([Log06], Corollary 3.16), so for any $\theta \in \Theta$ there is at least one normalized gnat-family maximizing $w_{\theta}$.

Proposition 6. Let $\mathcal{M}$ be any family which maximizes $w_{\theta}(\mathcal{M})$. Then for any point $y \in U$ the fiber of $\mathcal{M}$ at $y$ is a $\theta$-semistable $G$-constellation. If, moreover, $\theta$ is generic, then such family $\mathcal{M}$ is unique.

Proof. Write $\mathcal{M}$ as $\bigoplus \mathcal{L}\left(-M_{\chi}\right)$. Suppose that the fiber of $\mathcal{M}$ is not $\theta$-semistable at some $y \in U$. Denote this fiber by $A$, its decomposition into irreducible representations by $\bigoplus_{\chi \in G^{\vee}} A_{\chi}$ and the corresponding representation of the McKay quiver by $\left\{\alpha_{q}\right\}$. As $A$ isn't $\theta$-semistable there exists a non-empty proper subset $I$ of $G^{\vee}$ such that $A^{\prime}=\bigoplus_{\chi \in I} A_{\chi}$ is a sub- $R \rtimes G$-module of $A$ and $\theta\left(A^{\prime}\right)<0$. Denote by $J$ the complement $G^{\vee} \backslash I$. Denote by $Q_{I \rightarrow J}$ the subset $\left\{q \in Q_{1} \mid t q \in\right.$ $I, h q \in J\}$ of the arrow set $Q_{1}$ of the McKay quiver and similarly for $Q_{J \rightarrow I}$, $Q_{I \rightarrow I}, Q_{J \rightarrow J}$. Then $A^{\prime}$ being closed under the action of $R$ implies that for any $q \in Q_{I \rightarrow J}$ the map $\alpha_{q}$ is a zero map. Which by Proposition 5 implies $y \in B_{q}$.

The support of each $M_{\chi}$ consists only of the prime divisors in $\mathbb{E}([\log 06]$, Prop. 3.14 and 3.15). The same is true of the principal divisors $\left(x_{i}\right)$ for their images in $\mathbb{C}^{n} / G$ are the coordinate hyperplanes $x_{i}^{|G|}=0$. Therefore, by their defining equation (4.3), the support of each of the divisors of zeroes $B_{q}$ of $\mathcal{M}$ consists also only of the prime divisors in E. As $y$ lies on all $B_{q}$ with $q \in Q_{I \rightarrow J}$, $y$ must lie on at least one divisor in $\mathfrak{E}$. But, as $y \in U, y$ also lies on at most one divisor in $\mathfrak{E}$. Denote this unique divisor by $E$, then

$$
q \in Q_{I \rightarrow J} \Rightarrow E \subset B_{q} .
$$

Define a new $G$-Weil divisor set $\left\{M_{\chi}^{\prime}\right\}$ by setting $M_{\chi}^{\prime}$ to be $M_{\chi}$ if $\chi \in I$ and $M_{\chi}+E$ if $\chi \in J$. Then divisors $\left\{B_{q}^{\prime}\right\}$ defined from $\left\{M_{\chi}^{\prime}\right\}$ by equations (4.3) can be expressed as

$$
B_{q}^{\prime}=\left\{\begin{array}{ll}
B_{q} & \text { if } q \in Q_{I \rightarrow I}, Q_{J \rightarrow J} \\
B_{q}+E & \text { if } q \in Q_{J \rightarrow I} \\
B_{q}-E & \text { if } q \in Q_{I \rightarrow J}
\end{array} .\right.
$$


Since $\left\{B_{q}\right\}$ are all effective (4.8) and (4.7) imply that $\left\{B_{q}^{\prime}\right\}$ are also all effective. Therefore $\bigoplus \mathcal{L}\left(-M_{\chi}^{\prime}\right)$ is a normalized gnat-family. But

$$
w_{\theta}\left(\mathcal{M}^{\prime}\right)=w_{\theta}(\mathcal{M})+\sum_{\chi \in J} \theta(\chi)
$$

which contradicts the maximality of $w_{\theta}(\mathcal{M})$ since $\sum_{\chi \in J} \theta(\chi)=-\theta\left(A^{\prime}\right)>0$.

For the second claim let $\mathcal{N}=\bigoplus \mathcal{L}\left(-N_{\chi}\right)$ be another normalized family $\theta$-semistable over $U$. Let $B_{q}^{\prime}$ be divisors of zeroes of $\mathcal{N}$. Then

$$
B_{q}-B_{q}^{\prime}=\left(M_{t q}-N_{t q}\right)-\left(M_{h q}-N_{h q}\right)
$$

Take any $E^{\prime} \in \mathfrak{E}$ such that the sets $\left\{m_{\chi, E^{\prime}}\right\}$ and $\left\{n_{\chi, E^{\prime}}\right\}$ of the coefficients of $E^{\prime}$ in $\left\{M_{\chi}\right\}$ and $\left\{N_{\chi}\right\}$ are distinct. Then $J^{\prime}=\left\{\chi \in G^{\vee} \mid n_{\chi, E^{\prime}}>m_{\chi, E^{\prime}}\right\}$ is a non-empty proper subset of $G^{\vee}$. Denote by $I^{\prime}$ its complement. For any $q \in Q_{I^{\prime} \rightarrow J^{\prime}}$ the coefficient of $E^{\prime}$ in the RHS of (4.10) is strictly positive. As $B_{q}^{\prime}$ is effective we conclude that $q \in Q_{I^{\prime} \rightarrow J^{\prime}}$ implies $E^{\prime} \subset B_{q}$. So for any $y \in E^{\prime}$ the restriction $\left.\left(\bigoplus_{\chi \in I^{\prime}} \mathcal{L}\left(M_{\chi}\right)\right)\right|_{y}$ is a sub- $R \rtimes G$-module of $\mathcal{M}_{\mid y}$. But as $\mathcal{M}$ is $\theta$-semistable on $U$ and as $U \cap E^{\prime} \neq \emptyset$ we must have $\sum_{\chi \in I^{\prime}} \theta(\chi) \geq 0$. Similarly if $q \in Q_{J^{\prime} \rightarrow I^{\prime}}$, then the RHS of (4.10) is strictly negative, so $E^{\prime} \subset B_{q}^{\prime}$ and $\theta$-semistability of $\mathcal{N}$ implies $\sum_{\chi \in J^{\prime}} \theta(\chi)=-\sum_{\chi \in I^{\prime}} \theta(\chi) \geq 0$. Therefore $\sum_{\chi \in I^{\prime}} \theta(\chi)=0$ and $\theta$ is not generic.

The fine moduli space $M_{\theta}$ of $\theta$-stable $G$-constellations can be constructed via GIT theory, together with the universal family $\mathcal{M}_{\theta}$. The Hilbert-Chow morphism $\pi_{\theta}$ of $\mathcal{M}_{\theta}$ is projective. As the universal family is defined up to an equivalence of families, that is up to a twist by a line bundle, we can assume $\mathcal{M}_{\theta}$ to be normalised.

Assume for the rest of this section that $n=3$. If $\theta$ is generic, then $M_{\theta}$ is a projective crepant resolution of $\mathbb{C}^{3} / G$ and $\mathcal{M}_{\theta}$ is everywhere orthogonal in all degrees. As any two crepant resolutions of a canonical treefold are connected by a chain of flops, $M_{\theta}$ and $Y$ are isomorphic outside of a codimension 2 subset. The maps $Y \stackrel{\pi}{\rightarrow} \mathbb{C}^{3} / G$ and $M_{\theta} \stackrel{\pi_{\theta}}{\longrightarrow} \mathbb{C}^{3} / G$ fix a choice of a birational isomorphism between $Y$ and $M_{\theta}$. This, as described in Section 4.4, defines a notion of direct transforms between $Y$ and $M_{\theta}$.

Corollary 3. Let $\theta \in \Theta$ be generic. Let $\mathcal{M}$ be the unique normalized gnatfamily on $Y$ which maximizes the map $w_{\theta}$. Then $\mathcal{M}$ is isomorphic to the direct transform of $\mathcal{M}_{\theta}$ from $M_{\theta}$ to $Y$.

Proof. By the first claim of Proposition $6, \mathcal{M}$ is $\theta$-stable on $U$. So, by its definition, is the direct transform of $\mathcal{M}_{\theta}$ to $Y$. Hence, by the second claim of Proposition $6, \mathcal{M}$ and the direct transform of $\mathcal{M}_{\theta}$ must be isomorphic. 


\section{Non-projective example}

In this section we give an application of the Theorem 1 whereby we construct explicitly a derived McKay correspondence for a choice of an abelian $G \subset$ $\mathrm{SL}_{3}(\mathbb{C})$ and of a non-projective crepant resolution $Y$ of $\mathbb{C}^{3} / G$.

\subsection{The group}

We set the group $G$ to be $\frac{1}{6}(1,1,4) \oplus \frac{1}{2}(1,0,1)$. That is, the image in $\mathrm{SL}_{3}(\mathbb{C})$ of the product $\mu_{6} \times \mu_{2}$ of groups of 6 th and 2 nd roots of unity, respectively, under the embedding:

$$
\left(\xi_{1}, \xi_{2}\right) \mapsto\left(\begin{array}{ccc}
\xi_{1} \xi_{2} & & \\
& \xi_{1} & \\
& & \xi_{1}^{4} \xi_{2}
\end{array}\right)
$$

We denote by $\chi_{i, j}$ the character of $G$ induced by $\left(\xi_{1}, \xi_{2}\right) \mapsto \xi_{1}^{i} \xi_{2}^{j}$.

Calculating the McKay quiver of $G$ (cf. Section 4.1), we obtain:

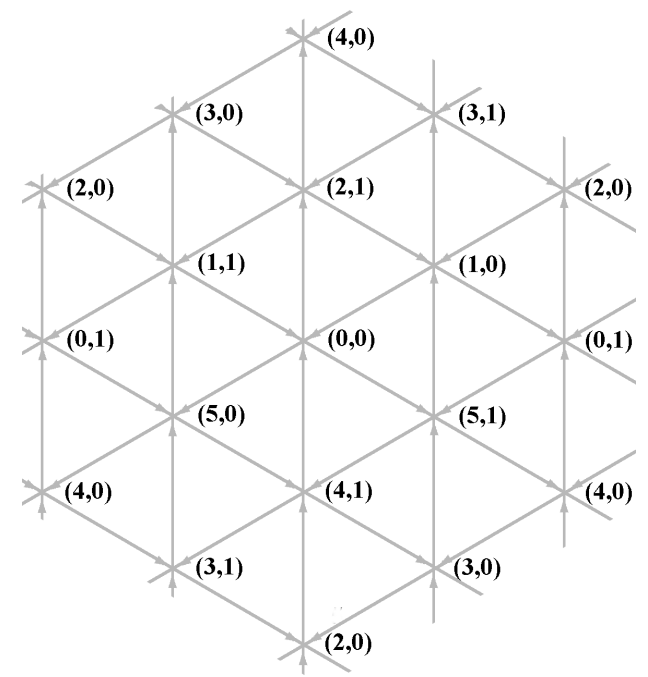

Figure 2

The way we've chosen to depict the McKay quiver reflects the fact that it has a universal cover quiver naturally embedded into $\mathbb{R}^{2}$. This point of view will not be essential for our argument but a curious reader should consult [CI04], Section 10.2 and [Log04], Section 6.4. 


\subsection{The resolution}

We define the crepant resolution $Y$ of $\mathbb{C}^{3} / G$ using methods of toric geometry. For the specifics related to $G$-constellations see $[\log 03]$, Section 3.

We define the relevant notation. The embedding (5.1) defines a surjection of torii

$$
0 \longrightarrow G \longrightarrow\left(\mathbb{C}^{*}\right)^{3} \longrightarrow T \longrightarrow 0 .
$$

Applying $\operatorname{Hom}\left(\bullet, \mathbb{C}^{*}\right)$ to $(5.2)$ we obtain the character lattices of the torii:

$$
0 \longrightarrow M \longrightarrow \mathbb{Z}^{3} \stackrel{\rho}{\longrightarrow} G^{\vee} \longrightarrow 0 \text {. }
$$

Given any character $m=\left(k_{1}, k_{2}, k_{3}\right) \in \mathbb{Z}^{3}$ of $\left(\mathbb{C}^{*}\right)^{3}$ we denote by $x^{m}$ the Laurent monomial $x_{1}^{k_{1}} x_{2}^{k_{2}} x_{3}^{k_{3}}$ in $R$. Applying $\operatorname{Hom}(\bullet, \mathbb{Z})$ to $(5.3)$ we obtain the dual lattices

$$
0 \longrightarrow\left(\mathbb{Z}^{3}\right)^{\vee} \longrightarrow N \longrightarrow \operatorname{Ext}^{1}\left(G^{\vee}, \mathbb{Z}\right) \longrightarrow 0
$$

Let $e_{1}, e_{2}, e_{3}$ be the basis of $\left(\mathbb{Z}^{3}\right)^{\vee}$ dual to $x_{1}, x_{2}, x_{3}$. The dual lattice $N$ is generated over $\left(\mathbb{Z}^{3}\right)^{\vee}$ by $\frac{1}{6}(1,1,4)$ and $\frac{1}{2}(1,0,1)$. The quotient space $\mathbb{C}^{3} / G$ is the toric variety given by a single cone $\sigma_{\geq 0}=\sum \mathbb{R}_{\geq 0} e_{i}$ in $N$. Let $Y$ be the toric variety whose fan $\mathfrak{F}$ in $N$ is the subdivision of $\sigma_{\geq 0}$ which triangulates the junior simplex $\Delta=\left\{\left(k_{1}, k_{2}, k_{3}\right) \in \sigma_{\geq 0} \mid \sum k_{i}=1\right\}$ as depicted below

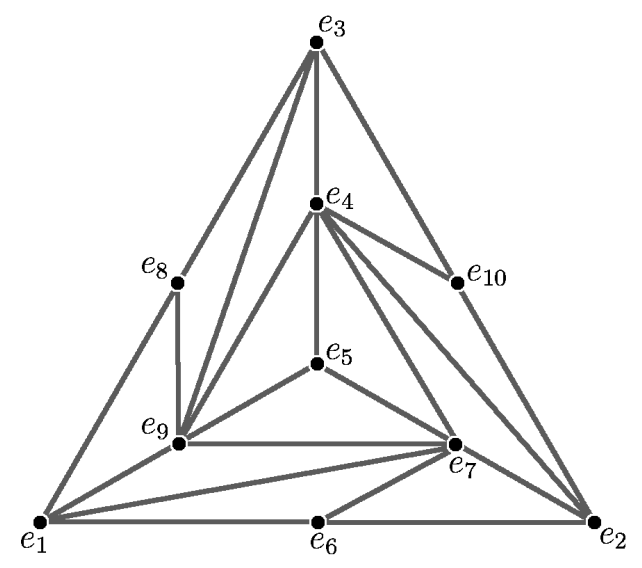

Figure 3

where by $e_{i}$ we denote the following elements of $N$

$$
\begin{array}{lll}
e_{1}=(1,0,0) & e_{2}=(0,1,0) & e_{3}=(0,0,1) \\
e_{4}=\frac{1}{6}(1,1,4) & e_{5}=\frac{1}{3}(1,1,1) & e_{6}=\frac{1}{2}(1,1,0) \\
e_{7}=\frac{1}{6}(1,4,1) & e_{8}=\frac{1}{2}(1,0,1) & e_{9}=\frac{1}{6}(4,1,1) \\
e_{10}=\frac{1}{2}(0,1,1) . & &
\end{array}
$$


Denote by $\pi$ the map $Y \rightarrow \mathbb{C}^{3} / G$ defined by the inclusion of $\mathfrak{F}$ into $\sigma \geq 0$. All the maximal cones of $\mathfrak{F}$ are basic in $N$, so $Y$ is smooth. The generators $e_{i}$ of the rays of $\mathfrak{F}$ lie in $\Delta$, so the map $\pi$ is crepant([Rei87], Prop. 4.8). Finally, the argument of [KKMSD73], Chapter III, §2E, Example 2 shows that $\pi$ is non-projective.

The quotient torus $T$ acts on $Y$ and to each $k$-dimensional cone $\sigma$ in $\mathfrak{F}$ corresponds a $(3-k)$-dimensional orbit of $T$. We denote it by $S_{\sigma}$ and denote by $E_{\sigma}$ the closure of $S_{\sigma}$, it is the union of all orbits $S_{\sigma^{\prime}}$ with $\sigma \subseteq \sigma^{\prime}$. For each cone $\left\langle e_{i}\right\rangle$ in the fan $\mathfrak{F}$, we denote by $S_{i}$ the codimension 1 orbit $S_{\left\langle e_{i}\right\rangle}$ and by $E_{i}$ the divisor $E_{\left\langle e_{i}\right\rangle}$. Similarly we use $S_{i, j}$ and $E_{i, j}$ for the codimension 2 orbit $S_{\left\langle e_{i}, e_{j}\right\rangle}$ and the surface $E_{\left\langle e_{i}, e_{j}\right\rangle}$ and we use $E_{i, j, k}$ for the toric fixed point $E_{\left\langle e_{i}, e_{j}, e_{k}\right\rangle}$.

\subsection{The family}

The map $Y \stackrel{\pi}{\rightarrow} \mathbb{C}^{3} / G$ defines the notion of $G$-Weil divisors on $Y$. Any normalized gnat-family on $Y \stackrel{\pi}{\rightarrow} \mathbb{C}^{3} / G$ is of the form $\bigoplus_{\chi \in G^{\vee}} \mathcal{L}\left(-D_{\chi}\right)$ for some $G$-Weil divisors $D_{\chi}$ with $D_{\chi_{0,0}}=0$. Moreover, as explained in [Log06], Section 3.5, there exists the maximal shift family $\oplus \mathcal{L}\left(-M_{\chi}\right)$ such that for any other normalized gnat-family $\oplus \mathcal{L}\left(-D_{\chi}\right)$ we have

$$
M_{\chi} \geq D_{\chi}
$$

for all $\chi \in G^{\vee}$. We denote this family by $\mathcal{F}$ and shall prove it to satisfy the assumptions of Corollary 1.

In the notation of Section 5.2 each divisor $M_{\chi}$ is of form $\sum q_{\chi, i} E_{i}$. The coefficients $q_{\chi, i}$ can be calculated via formula

$$
q_{\chi, i}=\inf \left\{e_{i}(m) \mid m \in \sigma_{\geq 0}^{\vee} \cap \rho^{-1}(\chi)\right\} .
$$

A detailed example of such calculation can be seen in [Log03], Example 4.21. In our case, we obtain $q_{\chi, i}$ to be:

\begin{tabular}{|c|c|c|c|c|c|c|c||c|c|c|c|c|c|c|c|c|}
\hline$\chi \backslash i$ & 4 & 5 & 6 & 7 & 8 & 9 & 10 & $\chi \backslash i$ & 4 & 5 & 6 & 7 & 8 & 9 & 10 \\
\hline$\chi_{0,0}$ & 0 & 0 & 0 & 0 & 0 & 0 & 0 & $\chi_{2,0}$ & $\frac{2}{6}$ & $\frac{4}{6}$ & 0 & $\frac{2}{6}$ & 0 & $\frac{2}{6}$ & 0 \\
$\chi_{4,0}$ & $\frac{4}{6}$ & $1 \frac{2}{6}$ & 0 & $\frac{4}{6}$ & 0 & $\frac{4}{6}$ & 0 & $\chi_{1,1}$ & $\frac{1}{6}$ & $\frac{2}{6}$ & $\frac{3}{6}$ & $\frac{1}{6}$ & $\frac{3}{6}$ & $\frac{4}{6}$ & 0 \\
$\chi_{1,0}$ & $\frac{1}{6}$ & $\frac{2}{6}$ & $\frac{3}{6}$ & $\frac{4}{6}$ & 0 & $\frac{1}{6}$ & $\frac{3}{6}$ & $\chi_{4,1}$ & $\frac{4}{6}$ & $\frac{2}{6}$ & 0 & $\frac{1}{6}$ & $\frac{3}{6}$ & $\frac{1}{6}$ & $\frac{3}{6}$ \\
$\chi_{3,1}$ & $\frac{3}{6}$ & 1 & $\frac{3}{6}$ & $\frac{3}{6}$ & $\frac{3}{6}$ & 1 & 0 & $\chi_{3,0}$ & $\frac{3}{6}$ & 1 & $\frac{3}{6}$ & 1 & 0 & $\frac{3}{6}$ & $\frac{3}{6}$ \\
$\chi_{0,1}$ & 1 & 1 & 0 & $\frac{3}{6}$ & $\frac{3}{6}$ & $\frac{3}{6}$ & $\frac{3}{6}$ & $\chi_{5,1}$ & $\frac{5}{6}$ & $\frac{4}{6}$ & $\frac{3}{6}$ & $\frac{5}{6}$ & $\frac{3}{6}$ & $\frac{2}{6}$ & 0 \\
$\chi_{5,0}$ & $\frac{5}{6}$ & $\frac{4}{6}$ & $\frac{3}{6}$ & $\frac{2}{6}$ & 0 & $\frac{5}{6}$ & $\frac{3}{6}$ & $\chi_{2,1}$ & $\frac{2}{6}$ & $\frac{4}{6}$ & 0 & $\frac{5}{6}$ & $\frac{3}{6}$ & $\frac{5}{6}$ & $\frac{3}{6}$ \\
\hline
\end{tabular}


The principal $G$-Weil divisors $\left(x_{k}\right)$ can be calculated with a formula

$$
\left(x_{i}\right)=\frac{1}{12} \sum_{j=1}^{10} e_{j}\left(x_{i}^{12}\right) E_{j},
$$

cf. [Log03], Prop. 3.2. In our case we obtain:

$$
\begin{aligned}
& \left(x_{1}\right)=E_{1}+\frac{1}{6} E_{4}+\frac{1}{3} E_{5}+\frac{1}{2} E_{6}+\frac{1}{6} E_{7}+\frac{1}{2} E_{8}+\frac{4}{6} E_{9} \\
& \left(x_{2}\right)=E_{2}+\frac{1}{6} E_{4}+\frac{1}{3} E_{5}+\frac{1}{2} E_{6}+\frac{4}{6} E_{7}+\frac{1}{6} E_{9}+\frac{1}{2} E_{10} \\
& \left(x_{3}\right)=E_{3}+\frac{4}{6} E_{4}+\frac{1}{3} E_{5}+\frac{1}{6} E_{7}+\frac{1}{2} E_{8}+\frac{1}{6} E_{9}+\frac{1}{2} E_{10}
\end{aligned}
$$

Substituting the data of (5.9) and (5.7) into the formula (4.3) we calculate for every arrow of the McKay quiver its divisor of zeroes in $\mathcal{F}$ :

$$
\begin{array}{ll}
B_{\chi_{0,0}, 1}=E_{1} & B_{\chi_{1,1}, 1}=E_{1}+E_{4}+E_{5}+E_{6}+E_{7}+E_{8}+E_{9} \\
B_{\chi_{0,0}, 2}=E_{2} & B_{\chi_{1,1}, 2}=E_{2}+E_{6}+E_{7} \\
B_{\chi_{0,0}, 3}=E_{3} & B_{\chi_{1,1}, 3}=E_{3}+E_{4}+E_{8} \\
B_{\chi_{4,0}, 1}=E_{1} & B_{\chi_{1,0}, 1}=E_{1}+E_{6}+E_{9} \\
B_{\chi_{4,0}, 2}=E_{2} & B_{\chi_{1,0}, 2}=E_{2}+E_{4}+E_{5}+E_{6}+E_{7}+E_{9}+E_{10} \\
B_{\chi_{4,0}, 3}=E_{3} & B_{\chi_{1,0}, 3}=E_{3}+E_{4}+E_{10} \\
B_{\chi_{2,0}, 1}=E_{1}+E_{5}+E_{9} & B_{\chi_{1,1}, 1}=E_{1}+E_{8}+E_{9} \\
B_{\chi_{\chi, 0}, 2}=E_{2}+E_{5}+E_{7} & B_{\chi_{4,1}, 2}=E_{2}+E_{7}+E_{10} \\
B_{\chi_{\chi, 0}, 3}=E_{3}+E_{4}+E_{5} & B_{\chi_{4,1}, 3}=E_{3}+E_{4}+E_{5}+E_{7}+E_{8}+E_{9}+E_{10} \\
B_{\chi_{5,1}, 1}=E_{1}+E_{6}+E_{8}+E_{9} & B_{\chi_{3,1}, 1}=E_{1}+E_{6}+E_{8}+E_{9} \\
B_{\chi_{5,1}, 2}=E_{2}+E_{6} & B_{\chi_{3,1}, 2}=E_{2}+E_{5}+E_{6}+E_{7}+E_{9} \\
B_{\chi_{5,1}, 3}=E_{3}+E_{8} & B_{\chi_{3,1}, 3}=E_{3}+E_{4}+E_{5}+E_{8}+E_{9} \\
B_{\chi_{5,0}, 1}=E_{1}+E_{6} & B_{\chi_{3,0}, 1}=E_{1}+E_{5}+E_{6}+E_{7}+E_{9} \\
B_{\chi_{5,0}, 2}=E_{2}+E_{6}+E_{7}+E_{10} & B_{\chi_{3,0}, 2}=E_{2}+E_{6}+E_{7}+E_{10} \\
B_{\chi_{5,0}, 3}=E_{3}+E_{10} & B_{\chi_{3,0}, 3}=E_{3}+E_{4}+E_{5}+E_{7}+E_{10} \\
B_{\chi_{2,1}, 1}=E_{1}+E_{8} & B_{\chi_{0,1}, 1}=E_{1}+E_{4}+E_{5}+E_{8}+E_{9} \\
B_{\chi_{2,1}, 2}=E_{2}+E_{10} & B_{\chi_{0,1}, 2}=E_{2}+E_{4}+E_{5}+E_{7}+E_{10} \\
B_{\chi_{2,1}, 3}=E_{3}+E_{4}+E_{8}+E_{10} & B_{\chi_{0,1}, 3}=E_{3}+E_{4}+E_{8}+E_{10} .
\end{array}
$$

\subsection{A sample calculation}

Corollary 2 together with the table (5.10) are all that we need to check any two $G$-constellations in $\mathcal{F}$ for the degree 0 orthogonality. Below we give an example of a calculation which verifies that any point on the torus orbit $S_{8}$ and any point on the torus orbit $S_{1,7}$ are orthogonal in degree 0 in $\mathcal{F}$.

Let $a$ be any point of $S_{8}$. Then $a$ lies on no divisor $E_{i}$ other than $E_{8}$. Hence $a \in B_{q}$ if and only if $E_{8} \subset B_{q}$. Let $A$ be the fiber of $\mathcal{F}$ at $a$ and $\left\{\alpha_{q}\right\}$ be the corresponding representation of the McKay quiver. By Proposition 5 for any 
arrow $q$ the map $\alpha_{q}$ is a zero map if and only if $E_{8} \in B_{q}$. On Figure 4 we use the table (5.10) and mark all the zero-maps in $\left\{\alpha_{q}\right\}$ by drawing a line through the corresponding arrow of the McKay quiver. Similarly if $b$ is a point of $S_{1,7}$ then $b$ lies on no $E_{i}$ other than $E_{1}$ and $E_{7}$. Let $B$ be the fiber of $\mathcal{F}$ at $b$ and $\left\{\beta_{q}\right\}$ be the corresponding representation. As above $\beta_{q}$ is a zero-map if and only if either $E_{1}$ or $E_{7}$ belongs to $B_{q}$. On Figure 5 we mark all the zero-maps $\left\{\beta_{q}\right\}$.

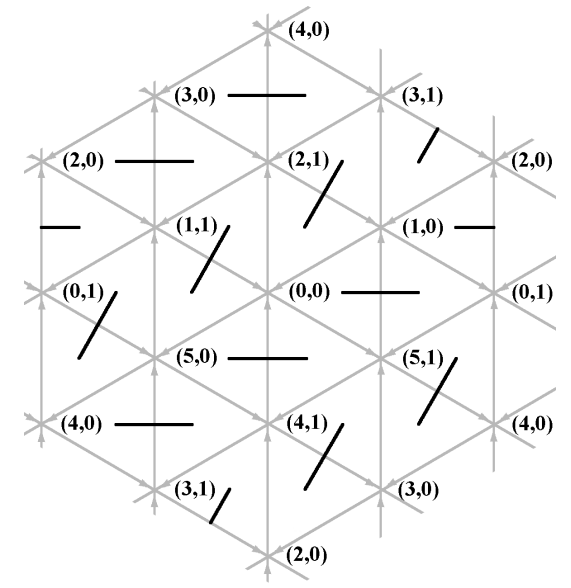

Figure 4

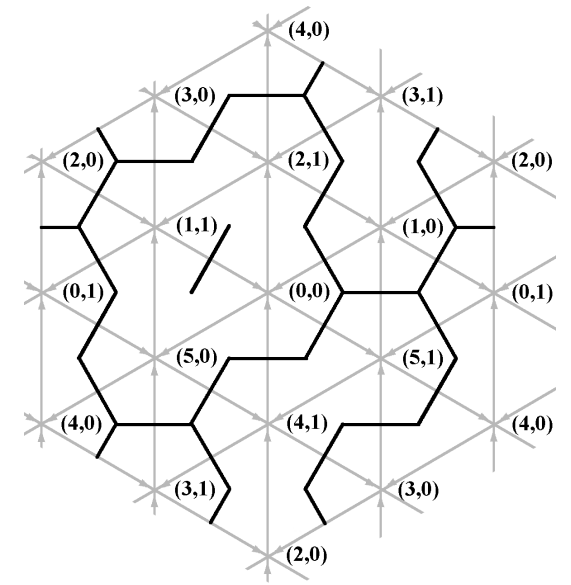

Figure 6

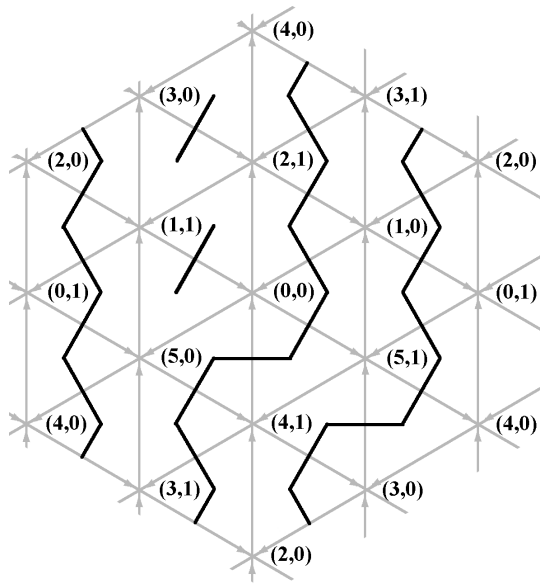

Figure 5

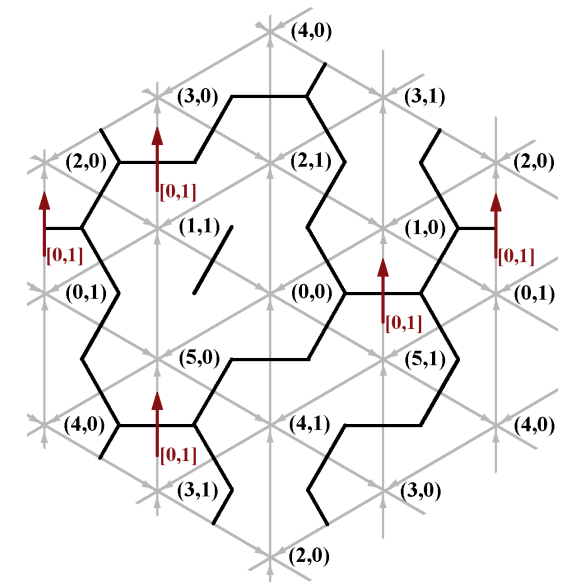

Figure 7

On Figure 6 we combine the markings of Figures 4 and 5 . The arrows left unmarked are the arrows of type $[1,1]$ with respect to the pair $A, B$ (Def. 7 ). It is clear that the components path-connected by $[1,1]$-arrows are: $\left\{\chi_{0,0}, \chi_{2,1}, \chi_{5,0}, \chi_{1,1}\right\}$, $\left\{\chi_{5,1}, \chi_{4,1}, \chi_{2,0}\right\},\left\{\chi_{1,0}, \chi_{3,1}\right\}$ and $\left\{\chi_{0,1}, \chi_{4,0}, \chi_{3,0}\right\}$. Now, with Cor. 2 in mind, we search the borders of these four regions for the $[1,0]$ and $[0,1]$-arrows. The $[1,0]$-arrows are the ones unmarked on Figure 4 but marked on Figure 5 and 
vice versa for $[0,1]$. On Figure 7 we've marked on the border of each region an incoming and an outgoing $[0,1]$-arrow. By Cor. 2 we see that $A$ and $B$ are orthogonal in degree 0.

\subsection{Final calculations}

We now claim that $\mathcal{F}$ is the direct transform of the universal family of $G$-clusters on $G$-Hilb $\left(\mathbb{C}^{3}\right)$. In the notation of Section 4.5 define $\theta_{+} \in \Theta$ by $\theta_{+}\left(\chi_{0,0}\right)=$ $1-|G|$ and $\theta_{+}(\chi)=1$ for $\chi \neq \chi_{0,0}$. Evidently $\theta_{+}$is generic. It follows from the original observation by Ito and Nakajima in [IN00], §3, that $G$-clusters can be identified with $\theta_{+}$-stable $G$-constellations, thus identifying $G$-Hilb $\left(\mathbb{C}^{3}\right)$ with the fine moduli space $M_{\theta_{+}}$. On the other hand, inequalities (5.5) imply that $\mathcal{F}$ maximizes $\omega_{\theta_{+}}$on $Y \stackrel{\pi}{\rightarrow} \mathbb{C}^{3} / G$. Hence, by Corollary $3, \mathcal{F}$ is the direct transform of $\mathcal{M}_{\theta_{+}}$from $G$-Hilb $\left(\mathbb{C}^{3}\right)$ to $Y$.

For a detailed description of an algorithm which allows one to calculate the toric fan of $G$-Hilb $\left(\mathbb{C}^{3}\right)$ see in [CR02]. For our group $G$ we obtain:

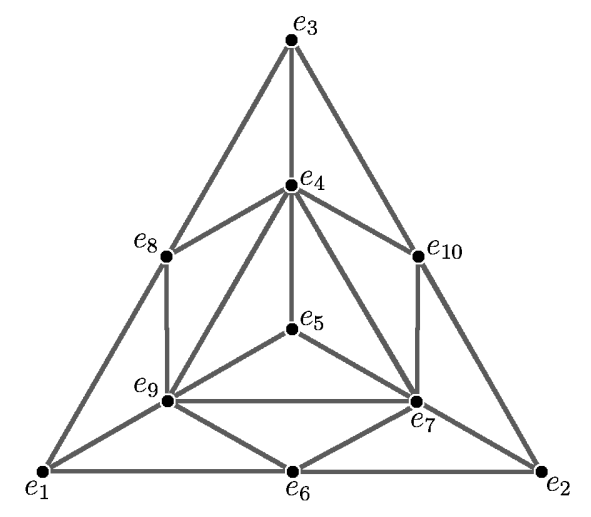

Figure 8

The general points of an exceptional surface $E_{i}$, as per the statement of Corollary 1 , are precisely the codimension 1 torus orbit $S_{i}$. Similarly, the general points of an exceptional curve $E_{i} \cap E_{j}$ are precisely the codimension 2 torus orbit $S_{i, j}$. Comparing Figure 8 with the fan of $Y$ on Figure 3 we see that the only codimension 1 or 2 torus orbits in $Y$ whose corresponding cones aren't also contained in the fan of $G$-Hilb $\left(\mathbb{C}^{3}\right)$ are $S_{1,7}, S_{2,4}$ and $S_{3,9}$. The argument in Section 4.4 reduces verifying that $\mathcal{F}$ satisfies the conditions of Corollary 1, to checking that each of these three orbits is orthogonal in degree 0 in $\mathcal{F}$ to every codimension 1 orbit $S_{i}$.

We claim that, in fact, it suffices to check it for just one of these orbits. Let $\phi$ be the rotation of the fan of $Y$ around the ray $e_{5}$ which rotates Figure 2 clockwise by $2 \pi / 3$. Let $\psi$ be the rotation of the plane containing the McKay quiver 
on the Figure 3 anti-clockwise by $2 \pi / 3$ with center at $\chi_{0,0}$. Observe that the permutation of the divisors $E_{i}$ defined by $\phi$ and the permutation of the arrows of the McKay quiver defined by $\psi$ leave the numerical data (5.10) of divisors of zeroes of $\mathcal{F}$ invariant ${ }^{1}$. It follows that the orthogonality calculation of Section 5.4 for any pair of torus orbits $S, S^{\prime}$ and the same calculation for $\phi(S), \phi\left(S^{\prime}\right)$ differ on Figures 4-7 only by a rotation by $\psi$. The claim now follows as the cones of $S_{1,7}, S_{2,4}$ and $S_{3,9}$ are permuted by $\phi$.

We choose to treat $S_{1,7}$. We repeat the calculation of Section 5.4 for $S_{1,7}$ and every other orbit $S_{i}$ and list below the analogues of Figure 7. From them, as elaborated in Section 5.4, the reader could readily ascertain the orthogonality in $\mathcal{F}$ of the torus orbits involved.

We conclude, by Corollary 1, that the integral transform $\Phi_{\mathcal{F}}\left(-\otimes \rho_{0}\right)$ is an equivalence of categories $D(Y) \rightarrow D^{G}\left(\mathbb{C}^{3}\right)$ and that a posteriori the family $\mathcal{F}$ is everywhere orthogonal in all degrees.

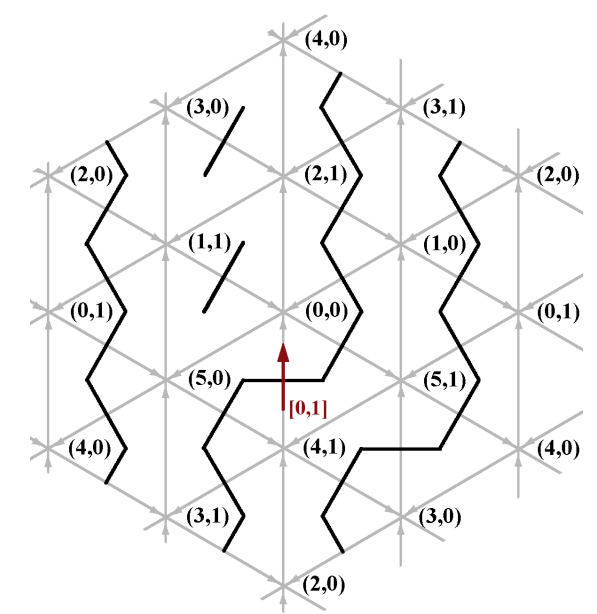

$\left(S_{1}, S_{1,7}\right)$ and $\left(S_{7}, S_{1,7}\right)$

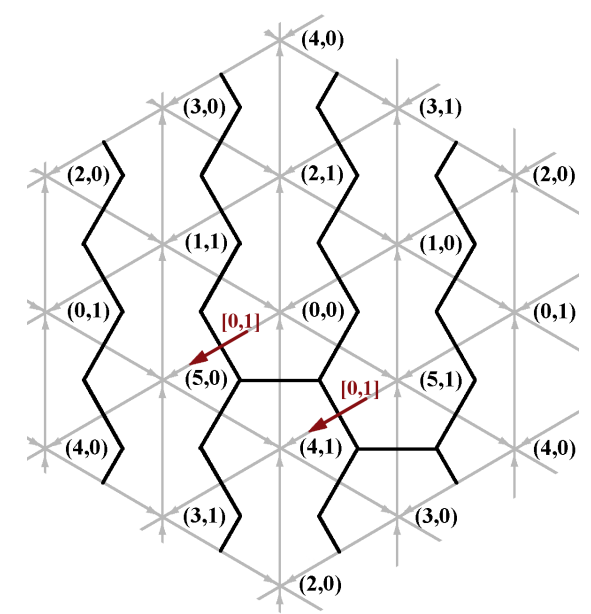

$\left(S_{2}, S_{1,7}\right)$

\footnotetext{
1 This invariance is a consequence of the fan of $Y$ being symmetric and of $\mathcal{F}$ being intrinsically defined as the maximal shift family.
} 


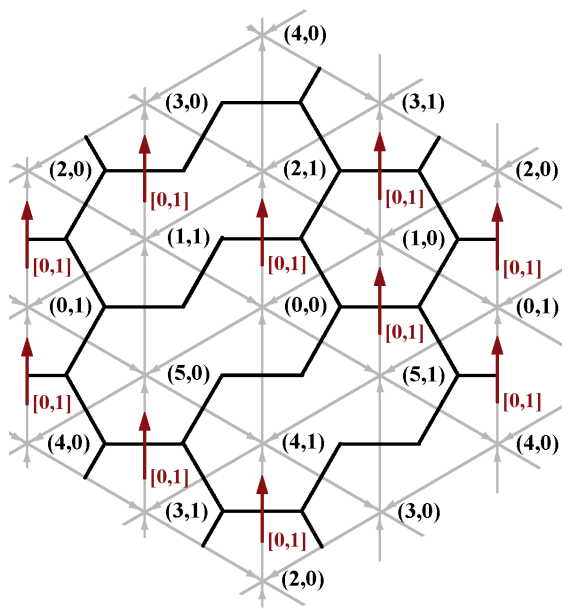

$\left(S_{3}, S_{1,7}\right)$
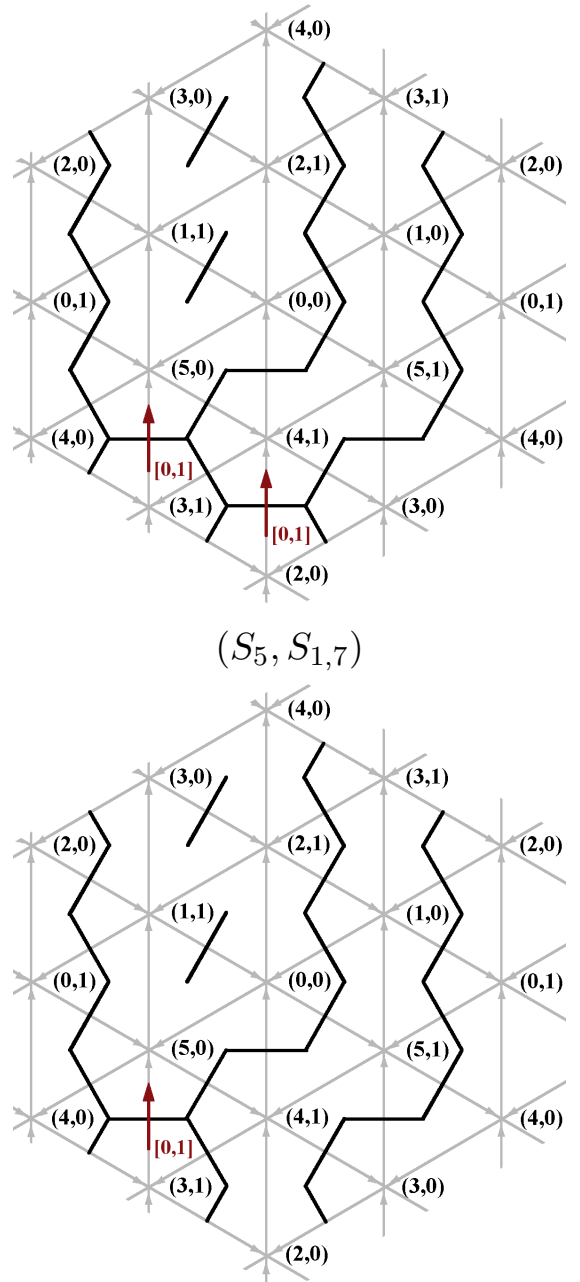

$\left(S_{9}, S_{1,7}\right)$

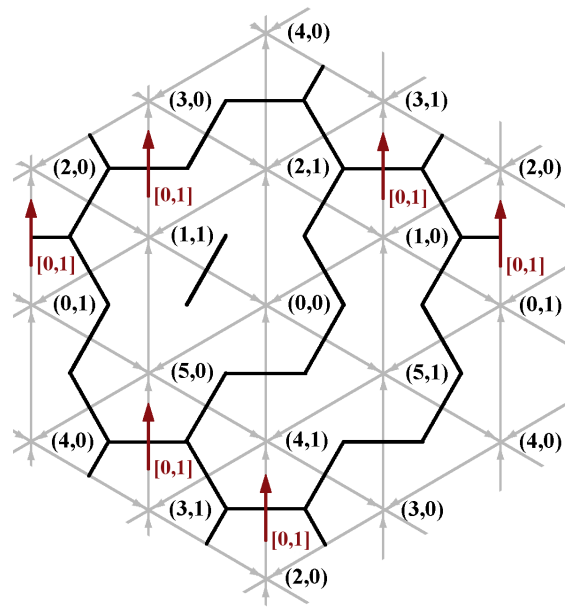

$\left(S_{4}, S_{1,7}\right)$

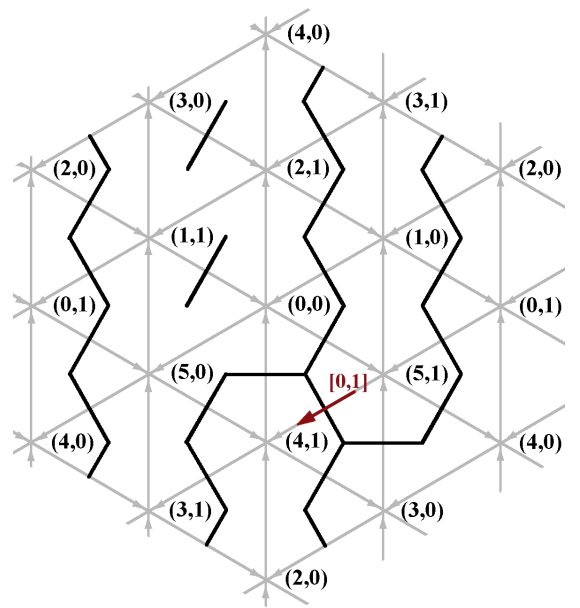

$\left(S_{6}, S_{1,7}\right)$

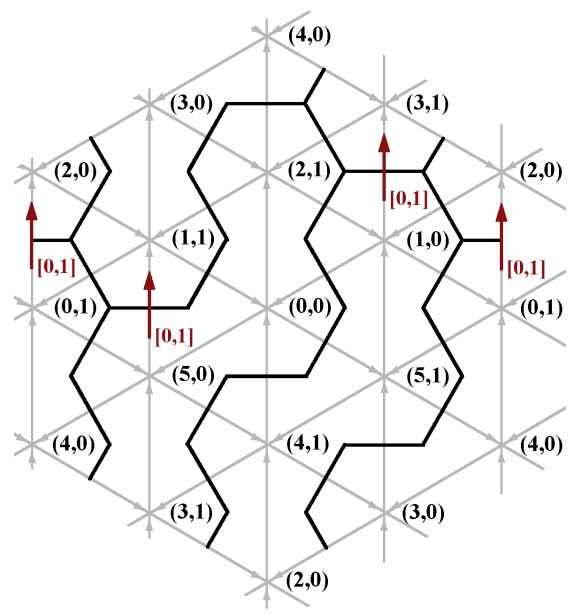

$\left(S_{10}, S_{1,7}\right)$ 


\section{References}

BK04. R. Bezrukavnikov and D. Kaledin, McKay equivalence for symplectic resolutions of singularities, Proc. Steklov Inst. Math 246 (2004), 13-33, math.AG/0401002.

BKR01. T. Bridgeland, A. King, and M. Reid, The McKay correspondence as an equivalence of derived categories, J. Amer. Math. Soc. 14 (2001), 535-554, math.AG/9908027.

BM02. T. Bridgeland and A. Macioca, Fourier-Mukai transforms for K3 and elliptic fibrations, J. Algebraic Geom. 11 (2002), no. 4, 629-657, math.AG/9908022.

BO95. A. Bondal and D. Orlov, Semi-orthogonal decompositions for algebraic varieties, preprint math.AG/950612, (1995).

Bri99. T. Bridgeland, Equivalence of triangulated categories and Fourier-Mukai transforms, Bull. London Math. Soc. 31 (1999), 25-34, math.AG/9809114.

Bri02. Stability conditions on triangulated categories, preprint math.AG/0504584, to appear in Annals of Mathematics, (2002).

CI04. A. Craw and A. Ishii, Flops of G-Hilb and equivalences of derived category by variation of GIT quotient, Duke Math J. 124 (2004), no. 2, 259-307, math.AG/0211360.

CMT05a. A. Craw, D. Maclagan, and R.R. Thomas, Moduli of McKay quiver representations I: the coherent component, preprint, (2005).

CMT05b. Moduli of McKay quiver representations II: Grobner basis techniques, preprint, (2005).

CR02. A. Craw and M. Reid, How to calculate $A$-Hilb $\mathbb{C}^{3}$, Seminaires et Congres 6 (2002), 129-154, math.AG/9909085.

Del66. P. Deligne, Cohomologie a support propre et construction du foncteur $f^{!}$, in "Residues and Duality", R. Hartshorne, Springer, 1966, pp. 404-421.

GD60. A. Grothendieck and J. Dieudonné, Éléments de géométrie algébrique I: Le langage des schémas., Publications mathématiques de l'I.H.É.S. 4 (1960), 5-228.

GD61. É_éments de géométrie algébrique III: Étude cohomologique des faisceaux cohérents. Première partie., Publications mathématiques de l'I.H.É.S. 11 (1961), 5-167.

GD63. É_éments de géométrie algébrique III: Étude cohomologique des faisceaux cohérents. Seconde partie., Publications mathématiques de l'I.H.É.S. 17 (1963), 591.

GD66. E Éléments de géométrie algébrique IV: Étude locale des schémas et des morphismes de schémas. Troisième partie., Publications mathématiques de l’I.H.É.S. 28 (1966), 5-255.

GM03. S.I. Gelfand and Yu. I. Manin, Methods of homological algebra, Springer, 2003.

GSV83. G. Gonzales-Sprinberg and J.-L. Verdier, Construction géométrique de la correspondance de McKay, Ann. sci. ENS 16 (1983), 409-449.

Har66. R. Hartshorne, Residues and duality, Springer-Verlag, 1966.

Huy06. D. Huybrechts, Fourier-Mukai transforms in algebraic geometry, Oxford University Press, 2006.

IN00. Y. Ito and H. Nakajima, McKay correspondence and Hilbert schemes in dimension three, Topology 39 (2000), no. 6, 1155-1191, math.AG/9803120.

Kaw05. Y. Kawamata, Log crepant birational maps and derived categories, J. Math. Sci. Univ. Tokyo 12 (2005), no. 2, 211-231, math.AG/0311139.

Kin94. A. King, Moduli of representations of finite-dimensional algebras, Quart. J. Math. Oxford 45 (1994), 515-530.

KKMSD73. G. Kempf, F. Knudsen, D. Mumford, and B. Saint-Donat, Toroidal embeddings I, Springer-Verlag, 1973. 
Kol89. J. Kollár, Flops, Nagoya Math J. 113 (1989), 15-36.

Kuz05. A. Kuznetsov, Homological projective duality, preprint math.AG/0507292, (2005).

KV98. M. Kapranov and E. Vasserot, Kleinian singularities, derived categories and hall algebras, preprint math.AG/9812016, (1998).

Log03. T. Logvinenko, Families of G-constellations over resolutions of quotient singularities, preprint math.AG/0305194, (2003).

Log04. _ Families of G-Constellations parametrised by resolutions of quotient singularities, Ph.D. thesis, University of Bath, 2004.

Log06. Natural G-constellation families, preprint math.AG/0601014, (2006).

Mat86. H. Matsumura, Commutative ring theory, Cambridge University Press, 1986.

McK80. J. McKay, Graphs, singularities and finite groups, Proc. Symp. Pure Math. 37 (1980), 183-186.

Muk81. S. Mukai, Duality between $D(X)$ and $D(\hat{X})$ with its application to Picard sheaves, Nagoya Math J 81 (1981), 153-175.

Nag. M. Nagata, Imbedding of an abstract variety in a complete variety, J. Math. Kyoto Uni. 2, no. 1.

Or197. D. Orlov, Equivalences of derived categories and K3 surfaces, J. Math. Sci. (NY) 84 (1997), no. 5, 1361-1381, math.AG/9606006.

Rei87. M. Reid, Young person's guide to canonical singularities, Proc. of Symposia in Pure Math. 46 (1987), 345-414.

Rei97. _ McKay correspondence, preprint math.AG/9702016, (1997).

Rob98. P. C. Roberts, Multiplicities and Chern classes in local algebra, Cambridge University Press, 1998.

Ser00. J.P. Serre, Local algebra, Springer, 2000. 